\title{
Solvability of a boundary value problem for singular multi-term fractional differential system with impulse effects
}

\author{
Xiaohui Yang ${ }^{1}$ and Yuji Liu ${ }^{2 *}$
}

${ }^{*}$ Correspondence:
liuyuji888@sohu.com
${ }^{2}$ Department of Mathematics,
Guangdong University of Finance
and Economics, Guangzhou,
510320, P.R. China
Full list of author information is
available at the end of the article

${ }^{\text {*Correspondence: }}$ liuyuji888@sohu.com ${ }^{2}$ Department of Mathematics, Guangdong University of Finance 510320, P.R. China available at the end of the article

\begin{abstract}
In this article, we first of all convert the boundary value problems for impulsive fractional differential equations to integral equations. Second, we construct a weighted function space and prove the completely continuous property of a nonlinear operator. Finally, we establish existence results for solutions of a boundary value problem for a nonlinear impulsive fractional differential system. Our analysis relies on the well-known Schauder fixed point theorem. An example is given to illustrate main results.
\end{abstract}

MSC: 92D25; 34A37; 34K15

Keywords: impulsive multi-term fractional differential system; boundary value problem; Schauder's fixed point theorem

\section{Introduction}

Fractional differential equations have many applications in modeling of physical and chemical processes $[1,2]$. In its turn, mathematical aspects of fractional differential equations and methods of their solutions were discussed by many authors; see the text books [3-5]. Existence of solutions or positive solutions of boundary value problems (BVPs for short) of fractional differential equations with or without impulse effects have been studied by many authors; see [6-16] and [17-19].

In recent years, some authors have studied the solvability or existence of positive solutions of BVPs of fractional differential systems [20-28]. In order to show motivations of this paper, we address some of them.

In [29], Su investigated the existence of positive solutions (continuous on $[0,1]$ ) of the following boundary value problem of nonlinear multi-term fractional differential system:

$$
\left\{\begin{array}{l}
D_{0+}^{\alpha} u+f\left(t, v(t), D_{0^{+}}^{p} v(t)\right)=0, \quad 0<t<1, \\
D_{0+}^{\beta} v+g\left(t, u(t), D_{0^{+}}^{q} u(t)\right)=0, \quad 0<t<1, \\
u(0)=0, \quad u(1)=0, \\
v(0)=0, \quad v(1)=0,
\end{array}\right.
$$

where $\alpha, \beta \in(1,2), D_{0+}$ is the Riemann-Liouville fractional derivative, $0<p<\beta-1,0<q<$ $\alpha-1, \gamma \eta^{\alpha-1}<1$, and $\gamma \eta^{\beta-1}<1, f, g:[0,1] \times \mathbb{R}^{+} \times \mathbb{R} \rightarrow \mathbb{R}$ are continuous functions.

(c) 2015 Yang and Liu. This article is distributed under the terms of the Creative Commons Attribution 4.0 International License (http://creativecommons.org/licenses/by/4.0/), which permits unrestricted use, distribution, and reproduction in any medium, provided you give appropriate credit to the original author(s) and the source, provide a link to the Creative Commons license, and indicate if changes were made. 
In [30], authors studied the existence of multiple positive solutions (continuous on $[0,1]$ ) of the following boundary value problem of $N$-dimension nonlinear fractional differential system:

$$
\left\{\begin{array}{l}
D_{0+}^{\alpha_{1}} u_{1}+f_{1}\left(t, u_{2}(t), D_{0^{+}}^{\mu_{1}} u_{2}(t)\right)=0, \quad 0<t<1, \\
\cdots, \\
D_{0+}^{\alpha_{N-1}} u_{N-1}+f_{N-1}\left(t, u_{N}(t), D_{0^{+}}^{\mu_{N}} u_{N}(t)\right)=0, \quad 0<t<1, \\
D_{0+}^{\alpha_{N}} u_{N}+f_{N}\left(t, u_{1}(t), D_{0^{+}}^{\mu_{N}} u_{1}(t)\right)=0, \quad 0<t<1, \\
u_{1}(0)=\cdots=u_{N}(0)=0 \\
u_{1}(1)=\cdots=u_{N}(1)=0
\end{array}\right.
$$

where $\alpha_{i} \in(1,2), D_{0+}$ is the Riemann-Liouville fractional derivative, $0<\mu_{i-1}<\alpha_{i}-1$ with $\mu_{0}=\mu_{N}, f_{i}:[0,1] \times \mathbb{R}^{+} \times \mathbb{R} \rightarrow \mathbb{R}(i=1,2, \ldots, N)$ are continuous functions.

In [31], the authors investigated the existence of positive solutions (continuous on $[0,1]$ ) of the following boundary value problem of nonlinear multi-term fractional differential system:

$$
\left\{\begin{array}{l}
D_{0_{+}}^{\alpha} u+f\left(t, v(t), D_{0^{+}}^{p} v(t)\right)=0, \quad 0<t<1, \\
D_{0+}^{\beta} v+g\left(t, u(t), D_{0^{+}}^{q} u(t)\right)=0, \quad 0<t<1 \\
u(0)=0, \quad u(1)=\gamma u(\eta), \\
v(0)=0, \quad v(1)=\gamma v(\eta),
\end{array}\right.
$$

where $\alpha, \beta \in(1,2), D_{0+}$ is the Riemann-Liouville fractional derivative, $0<p \leq \beta-1,0<$ $q \leq \alpha-1, \gamma \eta^{\alpha-1}<1$, and $\gamma \eta^{\beta-1}<1, f, g:[0,1] \times \mathbb{R}^{+} \times \mathbb{R} \rightarrow \mathbb{R}$ are continuous functions.

In [32], the authors studied the existence of solutions of the following four-point coupled boundary value problem for nonlinear fractional differential equation:

$$
\begin{cases}D_{0+}^{\alpha} u=f\left(t, u(t), D_{0^{+}}^{\alpha-1} u(t), v(t), D_{0^{+}}^{\beta-1} v(t)\right), & 0<t<1, \\ D_{0+}^{\beta} v=g\left(t, u(t), D_{0^{+}}^{\alpha-1} u(t), v(t), D_{0^{+}}^{\beta-1} v(t)\right), & 0<t<1, \\ I_{0^{+}}^{2-\alpha} u(0)=0, & u(1)=a v(\xi), \\ I_{0^{+}}^{2-\beta} v(0)=0, \quad v(1)=b u(\eta), & \end{cases}
$$

where $1<\alpha, \beta<2, D_{0^{+}}^{*}$, and $I_{0^{+}}^{*}$ are the standard Riemann-Liouville differentiation and integration, $f, g:[0,1] \times \mathbb{R}^{4} \rightarrow \mathbb{R}$ are continuous functions, $a, b \in \mathbb{R}, \xi, \eta \in(0,1)$ with $a b \xi^{\beta-1} \eta^{\alpha-1}=1$.

In [33], the following four-point boundary value problem of multi-term fractional differential system:

$$
\left\{\begin{array}{l}
D_{0+}^{\alpha} u=f\left(t, v(t), D_{0^{+}}^{m} v(t)\right), \quad 0<t<1, \\
D_{0+}^{\beta} v=g\left(t, u(t), D_{0^{+}}^{n} u(t)\right), \quad 0<t<1, \\
u(0)=\gamma u(\xi), \quad u(1)=\delta u(\eta) \\
v(0)=\gamma v(\xi), \quad v(1)=\delta v(\eta)
\end{array}\right.
$$

was studied, where $1<\alpha, \beta<2,0<m \leq \beta-1,0<n \leq \alpha-1, \gamma>0, \delta>0,0<\xi<\eta<1, D_{0^{+}}^{*}$ is the standard Riemann-Liouville differentiation, $f, g:[0,1] \times \mathbb{R}^{4} \rightarrow \mathbb{R}$ are continuous functions. 
In [34], the authors studied a coupled system of impulsive boundary value problems for nonlinear fractional order differential equations involving Caputo type fractional derivatives. Sufficient conditions for the existence and uniqueness of positive solutions were established by using Banach's fixed point theorem and Krasnoselskii's fixed point theorem.

In [35], the authors studied the following $2 m$-point boundary value problem for a coupled system of impulsive fractional differential equations at resonance:

$$
\left\{\begin{array}{l}
D_{0_{+}}^{\alpha} u=f\left(t, v(t), D_{0^{+}}^{p} v(t)\right), \quad 0<t<1, \\
D_{0^{\prime}}^{\beta} v=g\left(t, u(t), D_{0^{+}}^{q} u(t)\right), \quad 0<t<1, \\
\Delta u\left(t_{i}\right)=A_{i}\left(v\left(t_{i}\right), D_{0^{+}}^{p} v\left(t_{i}\right)\right), \quad \Delta D_{0^{+}}^{q} u\left(t_{i}\right)=B_{i}\left(v\left(t_{i}\right), D_{0^{+}}^{p} v\left(t_{i}\right)\right), \quad i=1,2, \ldots, k, \\
\Delta v\left(t_{i}\right)=C_{i}\left(u\left(t_{i}\right), D_{0^{+}}^{q} u\left(t_{i}\right)\right), \quad \Delta D_{0^{+}}^{p} v\left(t_{i}\right)=D_{i}\left(u\left(t_{i}\right), D_{0^{+}}^{q} u\left(t_{i}\right)\right), \quad i=1,2, \ldots, k, \\
D_{0^{+}}^{\alpha-1} u(0)=\sum_{i=1}^{m} a_{i} u\left(\xi_{i}\right), \quad u(1)=\sum_{i=1}^{m} b_{i} \eta_{i}^{2-\alpha} u\left(\eta_{i}\right), \\
D_{0^{+}}^{\beta-1} v(0)=\sum_{i=1}^{m} c_{i} v\left(\zeta_{i}\right), \quad v(1)=\sum_{i=1}^{m} d_{i} \theta_{i}^{2-\beta} v\left(\eta_{i}\right),
\end{array}\right.
$$

where $\alpha, \beta \in(1,2), \alpha-q \geq 1, \beta-p \geq 1,0<\xi_{1}<\cdots<\xi_{m}<1,0<\eta_{1}<\cdots<\eta_{m}<1,0<\zeta_{1}<$ $\cdots<\zeta_{m}<1$, and $0<\theta_{1}<\cdots<\theta_{m}<1, f, g:[0,1] \times \mathbb{R}^{2} \rightarrow \mathbb{R}$ satisfy Carathéodory conditions, $A_{i}, B_{i}, C_{i}, D_{i}: \mathbb{R} \times \mathbb{R} \rightarrow \mathbb{R}, \Delta w\left(t_{i}\right)=w\left(t_{i}^{+}\right)-w\left(t_{i}^{-}\right), \Delta D_{0^{+}}^{r} w\left(t_{i}\right)=D_{0^{+}}^{r} w\left(t_{i}^{+}\right)-D_{0^{+}}^{r} w\left(t_{i}^{-}\right)$with $w \in\{u, v\}$ and $r \in\{p, q\}, w\left(t_{i}^{+}\right)$and $w\left(t_{i}^{-}\right)$denote the right and left limits of $w(t)$ at $t=t_{i}$, respectively, and the fractional derivative is understood in the Riemann-Liouville sense. $k, m, a_{i}, b_{i}, c_{i}, d_{i}(i=1,2, \ldots, m)$ are fixed constants satisfying $\sum_{i=1}^{m} a_{i}=\sum_{i=1}^{m} b_{i}=\sum_{i=1}^{m} c_{i}=$ $\sum_{i=1}^{m} d_{i}=1$ and $\sum_{i=1}^{m} b_{i} \eta_{i}=\sum_{i=1}^{m} d_{i} \theta_{i}$. This system happens to be at resonance in the sense that the associated linear homogeneous coupled system

$$
\left\{\begin{array}{l}
D_{0^{+}}^{\alpha} u=0, \quad D_{0^{+}}^{\beta} v=0, \quad 0<t<1, \\
D_{0^{+}}^{\alpha-1} u(0)=\sum_{i=1}^{m} a_{i} D_{0^{+}}^{\alpha-1} u\left(\xi_{i}\right), \quad u(1)=\sum_{i=1}^{m} b_{i} \eta_{i}^{2-\alpha} u\left(\eta_{i}\right), \\
D_{0^{+}}^{\beta-1} v(0)=\sum_{i=1}^{m} c_{i} D_{0^{+}}^{\beta-1} v\left(\zeta_{i}\right), \quad v(1)=\sum_{i=1}^{m} d_{i} \theta_{i}^{2-\beta} v\left(\eta_{i}\right)
\end{array}\right.
$$

has $(u(t), v(t))=\left(h_{1} t^{\alpha-1}+h_{2} t^{\alpha-2}, h_{3} t^{\beta-1}+h_{4} t^{\beta-2}\right)\left(h_{i} \in R, i=1,2,3,4\right)$ as a nontrivial solution.

We find in the papers mentioned that $f$ in fractional differential equations is supposed to be continuous, the solutions obtained are also continuous on $[0,1]$. So it is interesting to study the solvability of boundary value problems of singular fractional differential equations and to obtain discontinuous solutions of this kind of problems.

In this paper, we study the existence of solutions of the following boundary value problems (BVP for short) for the multi-term impulsive fractional differential system:

$$
\left\{\begin{array}{l}
D_{0^{+}}^{\alpha} u(t)=p(t) f\left(t, v(t), D_{0^{+}}^{\theta} v(t), D_{0^{+}}^{\beta-1} v(t)\right), \quad t \in\left(t_{i}, t_{i+1}\right], i \in \mathbb{N}_{0}^{m}, \\
D_{0^{+}}^{\beta} v(t)=q(t) g\left(t, u(t), D_{0^{+}}^{\delta} u(t), D_{0^{+}}^{\alpha-1} u(t)\right), \quad t \in\left(t_{i}, t_{i+1}\right], i \in \mathbb{N}_{0}^{m}, \\
D_{0^{+}}^{\alpha-1} u(0)=0, \quad u(1)=0, \quad D_{0^{+}}^{\beta-1} v(0)=0, \quad v(1)=0, \\
\lim _{t \rightarrow t_{i}^{+}}\left(t-t_{i}\right)^{2-\alpha} u(t)=I_{1}\left(t_{i}, v\left(t_{i}\right), D_{0^{+}}^{\delta} v\left(t_{i}\right), D_{0^{+}}^{\beta-1} v\left(t_{i}\right)\right), \quad i \in \mathbb{N}_{1}^{m}, \\
\lim _{t \rightarrow t_{i}^{+}} D_{0^{+}}^{\alpha-1} u(t)-D_{0^{+}}^{\alpha-1} u\left(t_{i}\right)=J_{1}\left(t_{i}, v\left(t_{i}\right), D_{0^{+}}^{\delta} v\left(t_{i}\right), D_{0^{+}}^{\beta-1} v\left(t_{i}\right)\right), \quad i \in \mathbb{N}_{1}^{m}, \\
\lim _{t \rightarrow t_{i}^{+}}\left(t-t_{i}\right)^{2-\beta} v(t)=I_{2}\left(t_{i}, u\left(t_{i}\right), D_{0^{+}}^{\delta} u\left(t_{i}\right), D_{0^{+}}^{\alpha-1} u\left(t_{i}\right)\right), \quad i \in \mathbb{N}_{1}^{m}, \\
\lim _{t \rightarrow t_{i}^{+}} D_{0^{+}}^{\beta-1} v(t)-D_{0^{+}}^{\beta-1} v\left(t_{i}\right)=J_{2}\left(t_{i}, u\left(t_{i}\right), D_{0^{+}}^{\delta} u\left(t_{i}\right), D_{0^{+}}^{\alpha-1} u\left(t_{i}\right)\right), \quad i \in \mathbb{N}_{1}^{m},
\end{array}\right.
$$


where

(a) $\alpha, \beta \in(1,2), 0<\delta<\alpha-1,0<\theta<\beta-1, D_{a^{+}}^{b}$ is the Riemann-Liouville fractional derivative of order $b>0$ with the start point $a \in R$, see Definition 2.2,

(b) $m$ is a positive integer, $0=t_{0}<t_{1}<t_{2}<\cdots<t_{m}<t_{m+1}=1, \mathbb{N}_{0}^{m}=\{0,1,2, \ldots, m\}$, and $\mathbb{N}_{1}^{m}=\{1,2, \ldots, m\}$

(c) $p, q \in C^{0}(0,1)$ there exists $k_{i}>-1, l_{i} \leq 0$ with $2+k_{i}+l_{i}>0, \alpha+l_{1}-\delta>0$, $\beta+l_{2}-\theta>0$ such that $|p(t)| \leq t^{k_{1}}(1-t)^{l_{1}}$ and $|q(t)| \leq t^{k_{2}}(1-t)^{l_{2}}$ for all $t \in(0,1)$,

(d) $f:(0,1) \times \mathbb{R}^{3} \rightarrow \mathbb{R}$ is a $(\theta, \beta ; p)$-Carathéodory function, $g:(0,1) \times \mathbb{R}^{3} \rightarrow \mathbb{R}$ a $(\delta, \alpha ; q)$-Carathéodory function, see Definition $2.3, I_{1}, J_{1}:\left\{t_{i}\right\} \times \mathbb{R}^{3} \rightarrow \mathbb{R}$ are $(\theta, \beta)$-Carathéodory functions, $I_{2}, J_{2}:\left\{t_{i}\right\} \times \mathbb{R}^{3} \rightarrow \mathbb{R}(\delta, \alpha)$-Carathéodory functions, see Definition 2.4.

A pair of functions $u, v:(0,1] \rightarrow \mathbb{R}$ is called a solution of (6) if

$$
\begin{aligned}
& \left.v\right|_{\left(t_{i}, t_{i+1}\right]},\left.D_{0^{+}}^{\theta} v\right|_{\left(_{i}, t_{i+1}\right]},\left.D_{0^{+}}^{\beta-1} v\right|_{\left(t_{i}, t_{i+1}\right]} \in C^{0}\left(t_{i}, t_{i+1}\right], \quad i \in \mathbb{N}_{0}^{m}, \\
& \left.u\right|_{\left(t i_{i}, t_{i+1}\right]},\left.D_{0^{+}}^{\delta} u\right|_{\left(t_{i}, t_{i+1}\right]},\left.D_{0^{+}}^{\alpha-1} u\right|_{\left(t_{i}, t_{i+1}\right]} \in C^{0}\left(t_{i}, t_{i+1}\right], \quad i \in \mathbb{N}_{0}^{m}, \\
& \lim _{t \rightarrow t_{i}^{+}}\left(t-t_{i}\right)^{2-\beta} v(t), \lim _{t \rightarrow t_{i}^{+}}\left(t-t_{i}\right)^{2+\theta-\beta} D_{0^{+}}^{\theta} v(t), \lim _{t \rightarrow t_{i}^{+}} D_{0^{+}}^{\beta-1} v(t) \text { are finite, } \quad i \in \mathbb{N}_{0}^{m}, \\
& \lim _{t \rightarrow t_{i}^{+}}\left(t-t_{i}\right)^{2-\alpha} u(t), \lim _{t \rightarrow t_{i}^{+}}\left(t-t_{i}\right)^{2+\delta-\alpha} D_{0^{+}}^{\delta} u(t), \lim _{t \rightarrow t_{i}^{+}} D_{0^{+}}^{\alpha-1} u(t) \text { are finite, } \quad i \in \mathbb{N}_{0}^{m},
\end{aligned}
$$

$D_{0^{+}}^{\alpha} u$ and $D_{0^{+}}^{\beta} v$ are an $\alpha$-well integrable function and a $\beta$-well integrable function, respectively, and all equations in (6) are satisfied. Here the definitions of an $\alpha$-well integrable function and a $\beta$-well integrable function may be found in Definition 2.5.

The purpose of this paper is to obtain the results on the existence of solutions of BVP (6) by using the Schauder fixed point theorem [16] under some suitable assumptions. The solutions obtained may be discontinuous on $[0,1]$.

The main features of our paper are as follows. First of all, compared with the well-known papers [29-33], we construct a new Banach space and establish the existence results of solutions of (6). Second, boundary conditions are different from the known ones and impulse effects are imposed. Third, boundary conditions and impulse effects in (6) imply that solutions obtained in this paper are continuous on $\left(t_{i}, t_{i+1}\right]\left(i \in \mathbb{N}_{0}^{m}\right)$ but they may be unbounded on $(0,1)$. Finally, both $p f:(t, x, y) \mapsto p(t) f(t, x, y)$ and $q g:(t, x, y) \mapsto q(t) g(t, x, y)$ may be singular at $t=0,1$ while in the well-known papers mentioned nonlinearities are supposed to be continuous.

The remainder of the paper is organized as follows: In Section 2, we present some preliminary results. In Section 3, the existence results for solutions of BVP (6) are established. Finally, in Section 4, we present an example to illustrate the main theorems.

\section{Preliminary results}

In this section, we present some necessary definitions from the fractional calculus theory which can be found in the literature [1-5]. 
Definition 2.1 [1] The Riemann-Liouville fractional integral of order $\alpha>0$ of a function $h:(a,+\infty) \mapsto R$ is given by

$$
I_{a^{+}}^{\alpha} h(t)=\frac{1}{\Gamma(\alpha)} \int_{a}^{t}(t-s)^{\alpha-1} h(s) d s
$$

provided that the right-hand side exists.

Definition 2.2 [1] The Riemann-Liouville fractional derivative of order $\alpha>0$ of a function $h:(a,+\infty) \mapsto R$ is given by

$$
D_{a^{+}}^{\alpha} h(t)=\frac{1}{\Gamma(n-\alpha)} \frac{d^{n}}{d t^{n}} \int_{a}^{t} \frac{h(s)}{(t-s)^{\alpha-n+1}} d s,
$$

where $n-1 \leq \alpha<n$, provided that the right-hand side exists.

Lemma 2.1 (Schauder fixed point theorem) Let $\Omega$ be a closed convex subset of the Banach space X. Suppose $T: \Omega \mapsto \Omega$ and $T$ is compact (i.e., bounded sets in $\Omega$ are mapped into relatively compact sets). Then $T$ has a fixed point in $\Omega$.

Remark 2.1 Let $a<b$. From (2.106) in [5], we have $D_{a^{+}}^{\gamma} I_{a^{+}}^{\gamma} h(t)=h(t), t \in[a, b], \gamma>0$ if $f$ satisfies some suitable assumptions. For example we know $\left(\int_{a}^{t} h(s) d s\right)^{\prime}=h(t)$ for all continuous function $h:[a, b] \rightarrow \mathbb{R}$. From (2.108), in [5], for a function $h:[a, b] \mapsto \mathbb{R}$, if $D_{a^{+}}^{\alpha} h(t)$ $\left(D_{a^{+}}^{\delta} h(t), D_{a^{+}}^{\alpha-1} h(t)\right)$ is integrable, then we have $A, B, C, D \in \mathbb{R}$ such that

$$
\begin{aligned}
& I_{a^{+}}^{\alpha} D_{a^{+}}^{\alpha} h(t)=h(t)+A(t-a)^{\alpha-1}+B(t-a)^{\alpha-2}, \quad t \in(a, b], \\
& I_{a^{+}}^{\delta} D_{a^{+}}^{\delta} h(t)=h(t)+C(t-a)^{\delta-1}, \quad t \in(a, b], \\
& I_{a^{+}}^{\alpha-1} D_{a^{+}}^{\alpha-1} h(t)=h(t)+D(t-a)^{\alpha-2}, \quad t \in(a, b] .
\end{aligned}
$$

These results are generalizations of $\int h^{\prime}(s) d s=h(t)+C$ and $\left[\int h(t) d t\right]^{\prime}=h(t)$ if $h$ is an absolutely continuous function. If $h$ is a piecewise continuous function, what are the results? We give the following lemma.

Lemma 2.2 Let $\alpha \in(1,2)$. Suppose that $h:(0,1] \mapsto \mathbb{R}$ satisfies $\left.h\right|_{\left(t_{i}, t_{i+1}\right]}, D_{0^{+}}^{\alpha-1} h \in C^{0}\left(t_{i}, t_{i+1}\right]$ $\left(i \in \mathbb{N}_{0}^{m}\right), \lim _{t \rightarrow t_{i}^{+}}\left(t-t_{i}\right)^{2-\alpha} h(t)$, and $\lim _{t \rightarrow t_{i}^{+}} D_{0^{+}}^{\alpha-1} h(t)$ exist for all $i \in \mathbb{N}_{0}^{m}$. We can prove that there exist constants $c_{i, j} \in \mathbb{R}\left(i \in N_{0}^{m}, j=1,2\right)$ such that

$$
I_{0^{+}}^{\alpha} D_{0^{+}}^{\alpha} h(t)=h(t)+\sum_{j=0}^{i}\left(c_{1, j}\left(t-t_{j}\right)^{\alpha-1}+c_{2, j}\left(t-t_{j}\right)^{\alpha-2}\right), \quad t \in\left(t_{i}, t_{i+1}\right], i \in \mathbb{N}_{0}^{m},
$$

and

$$
D_{0^{+}}^{\alpha} I_{0^{+}}^{\alpha} h(t)=h(t), \quad t \in(0,1] .
$$

Proof Step 1. We first of all prove (10). Since $\lim _{t \rightarrow 0^{+}} t^{2-\alpha} h(t)=A_{0}$ exists, for any $\epsilon>0$, we have $A_{0}-\epsilon<t^{2-\alpha} h(t)<A_{0}+\epsilon$ for sufficiently small $t \in\left(0, t_{1}\right]$. Then there exists $A>0$ such 
that $|h(t)| \leq A t^{\alpha-2}$ for all $t \in\left(t_{0}, t_{1}\right]$. We have for $t \in\left(t_{0}, t_{1}\right]$

$$
\begin{aligned}
\left|\int_{0}^{t}(t-v)^{\alpha-1} h(v) d v\right| & \leq \int_{0}^{t}(t-v)^{\alpha-1}|h(v)| d v \\
& \leq A \int_{0}^{t}(t-v)^{\alpha-1} v^{\alpha-2} d v \quad \text { by } \frac{v}{t}=w \\
& =A t^{2 \alpha-2} \int_{0}^{1}(1-w)^{\alpha-1} w^{\alpha-2} d w .
\end{aligned}
$$

Then $\int_{0}^{t}(t-v)^{\alpha-1} h(v) d v$ is convergent for all $t \in\left(t_{0}, t_{1}\right]$. So for $t \in\left(0, t_{1}\right]$, we have

$$
\begin{aligned}
D_{0^{+}}^{\alpha} I_{0^{+}}^{\alpha} h(t) & =\frac{1}{\Gamma(2-\alpha) \Gamma(\alpha)}\left[\int_{0}^{t}(t-s)^{1-\alpha} \int_{0}^{s}(s-v)^{\alpha-1} h(v) d v d s\right]^{\prime \prime} \\
& =\frac{1}{\Gamma(2-\alpha) \Gamma(\alpha)}\left[\int_{0}^{t} \int_{s}^{t}(t-s)^{1-\alpha}(s-v)^{\alpha-1} d s h(v) d v\right]^{\prime \prime} \\
& =\frac{1}{\Gamma(2-\alpha) \Gamma(\alpha)}\left[\int_{0}^{t}(t-v) \int_{0}^{1}(1-w)^{1-\alpha} w^{\alpha-1} d w h(v) d v\right]^{\prime \prime} \\
& =\left[\int_{0}^{t}(t-v) h(v) d v\right]^{\prime \prime}=h(t) .
\end{aligned}
$$

Then $D_{0^{+}}^{\alpha} I_{0^{+}}^{\alpha} h(t)=h(t), t \in\left(t_{0}, t_{1}\right]$. For $t \in\left(t_{i+1}, t_{i+2}\right](i \geq 0)$, similar to above discussion, use that $\lim _{t \rightarrow t_{j}^{+}}\left(t-t_{j}\right)^{2-\alpha} h(t)$ exists, we know that $\int_{t_{j}}^{t_{j+1}}(t-v)^{\alpha-1} h(v) d v$ and $\int_{t_{i+1}}^{t}(t-v)^{\alpha-1} h(v) d v$ are convergent. So $\int_{0}^{t}(t-v)^{\alpha-1} h(v) d v$ is convergent. Then similarly to the above discussion $D_{0^{+}}^{\alpha} I_{0^{+}}^{\alpha} h(t)=h(t)$. The proof of $(10)$ is complete.

Step 2. We prove (9). Let $D_{0^{+}}^{\alpha} h(t)=H(t)$. Since $\lim _{t \rightarrow 0^{+}} t^{2-\alpha} h(t)=A_{0}$ exists, for any $\epsilon>0$, we have $A_{0}-\epsilon<t^{2-\alpha} h(t)<A_{0}+\epsilon$ for sufficiently small $t \in\left(0, t_{1}\right]$. Then for sufficiently small $t \in\left(0, t_{1}\right]$, we have

$$
\begin{aligned}
\left(A_{0}-\epsilon\right) \mathbf{B}(2-\alpha, \alpha-1) & \leq\left(A_{0}-\epsilon\right) \int_{0}^{t}(t-s)^{1-\alpha} s^{\alpha-2} d s<\int_{0}^{t}(t-s)^{1-\alpha} h(s) d s \\
& <\left(A_{0}+\epsilon\right) \int_{0}^{t}(t-s)^{1-\alpha} s^{\alpha-2} d s=\left(A_{0}+\epsilon\right) \mathbf{B}(2-\alpha, \alpha-1) .
\end{aligned}
$$

Then

$$
\begin{aligned}
\left(A_{0}-\epsilon\right) \mathbf{B}(2-\alpha, \alpha-1) & \leq \varlimsup_{t \rightarrow 0^{+}} \int_{0}^{t}(t-s)^{1-\alpha} h(s) d s \\
& \leq \varlimsup_{t \rightarrow 0^{+}} \int_{0}^{t}(t-s)^{1-\alpha} h(s) d s \leq\left(A_{0}+\epsilon\right) \mathbf{B}(2-\alpha, \alpha-1) .
\end{aligned}
$$

Let $\epsilon \rightarrow 0$, we get $\lim _{t \rightarrow 0^{+}} \int_{0}^{t}(t-s)^{1-\alpha} h(s) d s=A_{0} \mathbf{B}(2-\alpha, \alpha-1)$. Furthermore, since $\lim _{t \rightarrow 0^{+}} D_{0^{+}}^{\alpha-1} h(t)=B$ exists, $\lim _{t \rightarrow 0^{+}}\left(\int_{0}^{t}(t-s)^{1-\alpha} h(s) d s\right)^{\prime}=\Gamma(2-\alpha) B$ exists by Definition 2.2. So

$$
\begin{aligned}
I_{0^{+}}^{\alpha} H(t) & =I_{0^{+}}^{\alpha} D_{0^{+}}^{\alpha} h(t) \\
& =\frac{1}{\Gamma(2-\alpha) \Gamma(\alpha)} \int_{0}^{t}(t-s)^{\alpha-1}\left(\int_{0}^{s}(s-u)^{1-\alpha} h(u) d u\right)^{\prime \prime} d s
\end{aligned}
$$




$$
\begin{aligned}
& =\frac{1}{\Gamma(2-\alpha) \Gamma(\alpha)}\left[\int_{0}^{t}(t-s)^{\alpha-1} d\left(\int_{0}^{s}(s-u)^{1-\alpha} h(u) d u\right)^{\prime}\right] \\
& =\frac{1}{\Gamma(2-\alpha) \Gamma(\alpha)}\left[\left.(t-s)^{\alpha-1}\left(\int_{0}^{s}(s-u)^{1-\alpha} h(u) d u\right)^{\prime}\right|_{0} ^{t}\right. \\
& \left.+(\alpha-1) \int_{0}^{t}(t-s)^{\alpha-2}\left(\int_{0}^{s}(s-u)^{1-\alpha} h(u) d u\right)^{\prime} d s\right] \\
& =\frac{1}{\Gamma(2-\alpha) \Gamma(\alpha)}\left[-t^{\alpha-1} \lim _{s \rightarrow 0^{+}}\left(\int_{0}^{s}(s-u)^{1-\alpha} h(u) d u\right)^{\prime}\right. \\
& \left.+\left(\int_{0}^{t}(t-s)^{\alpha-1}\left(\int_{0}^{s}(s-u)^{1-\alpha} h(u) d u\right)^{\prime} d s\right)^{\prime}\right] \\
& =\frac{1}{\Gamma(2-\alpha) \Gamma(\alpha)}\left[-t^{\alpha-1} \lim _{s \rightarrow 0^{+}}\left(\int_{0}^{s}(s-u)^{1-\alpha} h(u) d u\right)^{\prime}\right. \\
& \left.+\left[\int_{0}^{t}(t-s)^{\alpha-1} d\left(\int_{0}^{s}(s-u)^{1-\alpha} h(u) d u\right)\right]^{\prime}\right] \\
& =\frac{1}{\Gamma(2-\alpha) \Gamma(\alpha)}\left[-t^{\alpha-1} \lim _{s \rightarrow 0^{+}}\left(\int_{0}^{s}(s-u)^{1-\alpha} h(u) d u\right)^{\prime}\right. \\
& -\left(t^{\alpha-1} \lim _{s \rightarrow 0^{+}} \int_{0}^{s}(s-u)^{1-\alpha} h(u) d u\right)^{\prime} \\
& \left.+(\alpha-1)\left(\int_{0}^{t}(t-s)^{\alpha-2} \int_{0}^{s}(s-u)^{1-\alpha} h(u) d u d s\right)^{\prime}\right] \\
& =\frac{1}{\Gamma(2-\alpha) \Gamma(\alpha)}\left[-t^{\alpha-1} \lim _{s \rightarrow 0^{+}}\left(\int_{0}^{s}(s-u)^{1-\alpha} h(u) d u\right)^{\prime}\right. \\
& -(\alpha-1) t^{\alpha-2} \lim _{s \rightarrow 0^{+}} \int_{0}^{s}(s-u)^{1-\alpha} h(u) d u \\
& \left.+(\alpha-1)\left(\int_{0}^{t} \int_{s}^{t}(t-s)^{\alpha-2}(s-u)^{1-\alpha} d s h(u) d u\right)^{\prime}\right] \quad \text { by } \frac{s-u}{t-u}=w \\
& =\frac{1}{\Gamma(2-\alpha) \Gamma(\alpha)}\left[-t^{\alpha-1} \Gamma(2-\alpha) B-(\alpha-1) t^{\alpha-2} A_{0} \mathbf{B}(2-\alpha, \alpha-1)\right. \\
& \left.+(\alpha-1)\left(\int_{0}^{t} \int_{0}^{1}(1-w)^{\alpha-2} w^{1-\alpha} d w h(u) d u\right)^{\prime}\right] \\
& =: c_{10} t^{\alpha-1}+c_{20} t^{\alpha-2}+h(t) \text {. }
\end{aligned}
$$

So

$$
I_{0^{+}}^{\alpha} D_{0^{+}}^{\alpha} h(t)=I_{0^{+}}^{\alpha} H(t)=h(t)+c_{10} t^{\alpha-1}+c_{20} t^{\alpha-2}, \quad t \in\left(0, t_{1}\right] .
$$

It follows from (11) that (9) holds for $i=0$. Now we suppose that (9) holds for $i=$ $0,1,2, \ldots, n<m$. We will prove that (9) holds for $i=n+1$. Then by the method of mathematical induction, we know (9) holds for all $i \in \mathbb{N}_{0}^{m}$.

In fact, suppose that

$$
I_{0^{+}}^{\alpha} D_{0^{+}}^{\alpha} h(t)=h(t)+\sum_{j=0}^{n}\left(c_{1, j}\left(t-t_{j}\right)^{\alpha-1}+c_{2, j}\left(t-t_{j}\right)^{\alpha-2}\right)+\Phi(t), \quad t \in\left(t_{n+1}, t_{n+2}\right] .
$$


Use the assumption, we get for $t \in\left(t_{n+1}, t_{n+2}\right]$

$$
\begin{aligned}
& H(t)=D_{0^{+}}^{\alpha} I_{0^{+}}^{\alpha} H(t)=D_{0^{+}}^{\alpha} I_{0^{+}}^{\alpha} D_{0^{+}}^{\alpha} h(t) \\
& =\frac{1}{\Gamma(2-\alpha)}\left[\int_{0}^{t}(t-s)^{1-\alpha} I_{0^{+}}^{\alpha} D_{0^{+}}^{\alpha} h(s) d s\right]^{\prime \prime} \\
& =\frac{1}{\Gamma(2-\alpha)}\left[\sum_{j=0}^{n} \int_{t_{j}}^{t_{j+1}}(t-s)^{1-\alpha} I_{0^{+}}^{\alpha} D_{0^{+}}^{\alpha} h(s) d s+\int_{t_{n+1}}^{t}(t-s)^{1-\alpha} I_{0^{+}}^{\alpha} D_{0^{+}}^{\alpha} h(s) d s\right]^{\prime \prime} \\
& =\frac{1}{\Gamma(2-\alpha)}\left[\sum_{j=0}^{n} \int_{t_{j}}^{t_{j+1}}(t-s)^{1-\alpha}\left(h(s)+\sum_{v=0}^{j}\left(c_{1, v}\left(s-t_{v}\right)^{\alpha-1}+c_{2, v}\left(s-t_{v}\right)^{\alpha-2}\right)\right) d s\right. \\
& \left.+\int_{t_{n+1}}^{t}(t-s)^{1-\alpha}\left(h(s)+\sum_{v=0}^{n}\left(c_{1, v}\left(s-t_{v}\right)^{\alpha-1}+c_{2, v}\left(s-t_{j}\right)^{\alpha-2}\right)+\Phi(s)\right) d s\right]^{\prime \prime} \\
& =D_{t_{n+1}^{+}}^{\alpha} \Phi(t)+D_{0^{+}}^{\alpha} h(t)+\frac{1}{\Gamma(2-\alpha)}\left[\sum_{j=0}^{n} \sum_{v=0}^{j} c_{1, v} \int_{t_{j}}^{t_{j+1}}(t-s)^{1-\alpha}\left(s-t_{v}\right)^{\alpha-1} d s\right. \\
& +\sum_{j=0}^{n} \sum_{v=0}^{j} c_{2, v} \int_{t_{j}}^{t_{j+1}}(t-s)^{1-\alpha}\left(s-t_{v}\right)^{\alpha-2} d s \\
& \left.+\sum_{v=0}^{n} c_{1, v} \int_{t_{n+1}}^{t}(t-s)^{1-\alpha}\left(s-t_{v}\right)^{\alpha-1} d s+\sum_{v=0}^{n} c_{2, v} \int_{t_{n+1}}^{t}(t-s)^{1-\alpha}\left(s-t_{j}\right)^{\alpha-2} d s\right]^{\prime \prime} \\
& =D_{t_{n+1}^{+}}^{\alpha} \Phi(t)+D_{0^{+}}^{\alpha} h(t)+\frac{1}{\Gamma(2-\alpha)}\left[\sum_{j=0}^{n} \sum_{v=0}^{j} c_{1, v}\left(t-t_{v}\right) \int_{\frac{t_{j}-t_{v}}{t-t_{v}}}^{\frac{t_{j+1}-t_{v}}{t-t_{v}}}(1-w)^{1-\alpha} w^{\alpha-1} d w\right. \\
& +\sum_{j=0}^{n} \sum_{v=0}^{j} c_{2, v} \int_{\frac{t_{j}-t_{v}}{t-t_{v}}}^{\frac{t_{j+1}-t_{v}}{t-t_{v}}}(1-w)^{1-\alpha} w^{\alpha-2} d w \\
& +\sum_{\nu=0}^{n} c_{1, \nu}\left(t-t_{\nu}\right) \int_{\frac{t_{n+1}-t_{v}}{t-t_{\nu}}}^{1}(1-w)^{1-\alpha} w^{\alpha-1} d w \\
& \left.+\sum_{\nu=0}^{n} c_{2, v} \int_{\frac{t_{n+1-t_{\nu}}}{t-t_{\nu}}}^{1}(1-w)^{1-\alpha} w^{\alpha-2} d w\right]^{\prime \prime} \\
& =D_{t_{n+1}^{+}}^{\alpha} \Phi(t)+D_{0^{+}}^{\alpha} h(t)+\frac{1}{\Gamma(2-\alpha)}\left[\sum_{\nu=0}^{n} c_{1, v}\left(t-t_{v}\right) \sum_{j=v}^{n} \int_{\frac{t_{j}-t_{v}}{t-t_{v}}}^{\frac{t_{j+1}-t_{\nu}}{t-t_{\nu}}}(1-w)^{1-\alpha} w^{\alpha-1} d w\right. \\
& +\sum_{v=0}^{n} c_{2, v} \sum_{j=v}^{n} \int_{\frac{t_{j}-t_{v}}{t-t_{v}}}^{\frac{t_{j+1}-t_{v}}{t-t_{v}}}(1-w)^{1-\alpha} w^{\alpha-2} d w \\
& +\sum_{\nu=0}^{n} c_{1, v}\left(t-t_{\nu}\right) \int_{\frac{t_{n+1}-t_{v}}{t-t_{v}}}^{1}(1-w)^{1-\alpha} w^{\alpha-1} d w \\
& \left.+\sum_{v=0}^{n} c_{2, v} \int_{\frac{t_{n+1-t_{v}}}{t-t_{v}}}^{1}(1-w)^{1-\alpha} w^{\alpha-2} d w\right]^{\prime \prime} \\
& =D_{t_{n+1}^{+}}^{\alpha} \Phi(t)+H(t)+\frac{1}{\Gamma(2-\alpha)}\left[\sum_{v=0}^{n} c_{1, v}\left(t-t_{v}\right) \int_{1}^{1}(1-w)^{1-\alpha} w^{\alpha-1} d w\right.
\end{aligned}
$$




$$
\begin{aligned}
& \left.+\sum_{\nu=0}^{n} c_{2, v} \int_{0}^{1}(1-w)^{1-\alpha} w^{\alpha-2} d w\right]^{\prime \prime} \\
= & D_{t_{n+1}^{+}}^{\alpha} \Phi(t)+H(t) .
\end{aligned}
$$

It follows that $D_{t_{n+1}^{+}}^{\alpha} \Phi(t)=0$ on $\left(t_{n+1}, t_{n+2}\right]$. Similarly to the proof of (11), there exist constants $c_{1, n+1}, c_{2, n+1} \in R$ such that $\Phi(t)=c_{1, n+1}\left(t-t_{n+1}\right)^{\alpha-1}+c_{2, n+1}\left(t-t_{n+1}\right)^{\alpha-2}$. Substituting $\Phi(t)$ into (12), we know that (9) holds for $i=n+1$. This completes the proof of (9).

Remark 2.2 Let $\delta \in(0,1)$. Suppose that $h:(0,1] \rightarrow \mathbb{R}$ satisfies $\left.h\right|_{\left(t_{i}, t_{i+1}\right]} \in C^{0}\left(t_{i}, t_{i+1}\right]$ $\left(i \in \mathbb{N}_{0}^{m}\right)$ and $\lim _{t \rightarrow t_{i}^{+}}\left(t-t_{i}\right)^{1-\delta} h(t)$ exists for all $i \in \mathbb{N}_{0}^{m}$. We can prove that there exist constants $c_{i} \in \mathbb{R}\left(i \in \mathbb{N}_{0}^{m}\right)$ such that

$$
\begin{aligned}
& I_{0^{+}}^{\delta} D_{0^{+}}^{\delta} h(t)=h(t)+\sum_{j=0}^{i} c_{j}\left(t-t_{j}\right)^{\delta-1}, \quad t \in\left(t_{i}, t_{i+1}\right], i \in \mathbb{N}_{0}^{m}, \\
& D_{0^{+}}^{\delta} I_{0^{+}}^{\delta} h(t)=h(t), \quad t \in(0,1] .
\end{aligned}
$$

Proof The proof is similar to that of Lemma 2.2 and is omitted.

Definition 2.3 Let $b>0, c, d \in R$ with $c<d$ be fixed. A function $h:(c, d) \rightarrow \mathbb{R}$ is called a $b$-well integrable function on $(c, d)$ if both $\int_{0}^{t}(t-s)^{b-1}|h(s)| d s$ and $\int_{0}^{t}(t-s)^{b-1} h(s) d s$ are convergent for all $t \in[c, d]$.

Definition 2.4 Let $b \in(1,2)$ and $a \in(0, b-1)$ and $\mu:(0,1) \rightarrow R . h:(0,1) \times \mathbb{R}^{3} \rightarrow \mathbb{R}$ is called a $(a, b ; \mu)$-Carathéodory function if

(i) $t \mapsto \mu(t) h\left(t, \frac{x_{1}}{\left(t-t_{i}\right)^{2-b}}, \frac{x_{2}}{\left(t-t_{i}\right)^{2+a-b}}, x_{3}\right)$ is a $b$-well integrable function on $(0,1]$ for every $\left(x_{1}, x_{2}, x_{3}\right) \in \mathbb{R}^{3}$,

(ii) $\left(x_{1}, x_{2}, x_{3}\right) \mapsto h\left(t, \frac{x_{1}}{\left(t-t_{i}\right)^{2-b}}, \frac{x_{2}}{\left(t-t_{i}\right)^{2+a-b}}, x_{3}\right)$ is continuous on $\mathbb{R}^{3}$ for each $t \in\left(t_{i}, t_{i+1}\right)$ $\left(i \in \mathbb{N}_{0}^{m}\right)$,

(iii) for each $r>0$, there exists $M_{r}>0$ such that $\left|x_{i}\right| \leq r(i=1,2,3)$ imply that

$$
\left|h\left(t, \frac{x_{1}}{\left(t-t_{i}\right)^{2-b}}, \frac{x_{2}}{\left(t-t_{i}\right)^{2+a-b}}, x_{3}\right)\right| \leq M_{r}, \quad t \in\left(t_{i}, t_{i+1}\right), i \in \mathbb{N}_{0}^{m} .
$$

Definition 2.5 Let $b \in(1,2)$ and $a \in(0, b-1) . I:\left\{t_{i}: i \in \mathbb{N}_{1}^{m}\right\} \times \mathbb{R}^{3} \rightarrow \mathbb{R}$ is a $(a, b)$ Carathéodory function if

(i) $\left(x_{1}, x_{2}, x_{3}\right) \mapsto I\left(t_{i}, \frac{x_{1}}{\left(t_{i}-t_{i-1}\right)^{2-b}}, \frac{x_{2}}{\left(t_{i}-t_{i-1}\right)^{2+a-b}}, x_{3}\right)$ is continuous on $\mathbb{R}^{3}$ for each $i \in N_{1}^{m}$,

(ii) for each $r>0$, there exists $M_{I, r}>0$ such that $\left|x_{i}\right| \leq r(i=1,2,3)$ imply that

$$
\left|I\left(t_{i}, \frac{x_{1}}{\left(t_{i}-t_{i-1}\right)^{2-b}}, \frac{x_{2}}{\left(t_{i}-t_{i-1}\right)^{2+a-b}}, x_{3}\right)\right| \leq M_{I, r}, \quad i \in \mathbb{N}_{1}^{m} .
$$

Choose

$$
\begin{aligned}
X_{\delta, \alpha}= & \left\{u:(0,1] \rightarrow R, u, D^{\delta} u, D^{\alpha-1} u \in C^{0}\left(t_{i}, t_{i+1}\right], i \in \mathbb{N}_{0}^{m},\right. \\
& \text { the following limits exist: } \lim _{t \rightarrow t_{i}^{+}}\left(t-t_{i}\right)^{2-\alpha} u(t), i \in \mathbb{N}_{0}^{m}, \\
& \left.\lim _{t \rightarrow t_{i}^{+}}\left(t-t_{i}\right)^{2+\delta-\alpha} D_{0^{+}}^{\delta} u(t), i \in \mathbb{N}_{0}^{m}, \lim _{t \rightarrow t_{i}^{+}} D_{0^{+}}^{\alpha-1} u(t), i \in \mathbb{N}_{0}^{m}\right\} .
\end{aligned}
$$


For $u \in X_{\delta, \alpha}$, define

$$
\|u\|=:\|u\|_{\delta, \alpha}=\max \left\{\begin{array}{l}
\sup _{t \in\left(t_{i}, t_{i+1}\right]}\left(t-t_{i}\right)^{2-\alpha}|u(t)| \\
\sup _{t \in\left(t_{i}, t_{i+1}\right]}\left(t-t_{i}\right)^{2+\delta-\alpha}\left|D_{0^{+}}^{\delta} u(t)\right|: i \in \mathbb{N}_{0}^{m} \\
\sup _{t \in\left(t_{i}, t_{i+1}\right]}\left|D_{0^{+}}^{\alpha-1} u(t)\right|
\end{array}\right\} .
$$

Lemma 2.3 $X_{\delta, \alpha}$ is a Banach space with the norm defined.

Proof In fact, it is easy to see that $X$ is a normed linear space with the norm $\|\cdot\|$. Let $\left\{x_{u}\right\}$ be a Cauchy sequence in $X_{\delta, \alpha}$. Then $\left\|x_{u}-x_{v}\right\| \rightarrow 0, u, v \rightarrow+\infty$. It follows that

$$
\begin{aligned}
& \sup _{t \in\left(t_{i}, t_{i+1}\right]}\left(t-t_{i}\right)^{2-\alpha}\left|x_{u}(t)-x_{v}(t)\right| \rightarrow 0, \quad v, u \rightarrow+\infty, i \in \mathbb{N}_{0}^{m}, \\
& \sup _{t \in\left(t_{i}, t_{i+1}\right]}\left(t-t_{i}\right)^{2+\delta-\alpha}\left|D_{0^{+}}^{\delta} x_{u}(t)-D_{0^{+}}^{\delta} x_{v}(t)\right| \rightarrow 0, \quad v, u \rightarrow+\infty, i \in \mathbb{N}_{0}^{m}, \\
& \sup _{t \in\left(t_{i}, t_{i+1}\right]}\left|D_{0^{+}}^{\alpha-1} x_{u}(t)-D_{0^{+}}^{\alpha-1} x_{v}(t)\right| \rightarrow 0, \quad v, u \rightarrow+\infty, i \in \mathbb{N}_{0}^{m} .
\end{aligned}
$$

Denote $x_{u, i}=\left.x_{u}\right|_{\left(t_{i}, t_{i+1}\right]}$. Since

$$
\lim _{t \rightarrow t_{i}^{+}}\left(t-t_{i}\right)^{2-\alpha} x_{u}(t), \lim _{t \rightarrow t_{i}^{+}}\left(t-t_{i}\right)^{2+\delta-\alpha} D_{0^{+}}^{\delta} x(t), \lim _{t \rightarrow t_{i}^{+}} D_{0^{+}}^{\alpha-1} x_{u}(t)
$$

exist, we know that $t \mapsto\left(t-t_{i}\right)^{2-\alpha} x_{u, i}(t)$ are continuous on $\left[t_{i}, t_{i+1}\right]$. Thus $t \mapsto\left(t-t_{i}\right)^{2-\alpha} x_{u, i}(t)$ are Cauchy sequences in $C\left[t_{i}, t_{i+1}\right]$. So $\left(t-t_{i}\right)^{2-\alpha} x_{u, i}(t)$ uniformly converges to some $x_{0, i}$ in $C\left[t_{i}, t_{i+1}\right]$ as $u \rightarrow+\infty$. It follows that

$$
\sup _{t \in\left[t_{i}, t_{i+1}\right]}\left|\left(t-t_{i}\right)^{2-\alpha} x_{u, i}(t)-x_{0, i}(t)\right| \rightarrow 0, \quad u \rightarrow+\infty, i \in \mathbb{N}_{0}^{m} .
$$

That is,

$$
\sup _{t \in\left[t_{i}, t_{i+1}\right]}\left(t-t_{i}\right)^{2-\alpha}\left|x_{u, i}(t)-\left(t-t_{i}\right)^{\alpha-1} x_{0, i}(t)\right| \rightarrow 0, \quad u \rightarrow+\infty, i \in \mathbb{N}_{0}^{m} .
$$

Let $x_{0}(t)=\left(t-t_{i}\right)^{\alpha-2} x_{0, i}(t)$ for $t \in\left(t_{i}, t_{i+1}\right]\left(i \in \mathbb{N}_{0}^{m}\right)$. It is easy to see that $x_{0} \in C^{0}\left(t_{i}, t_{i+1}\right]$

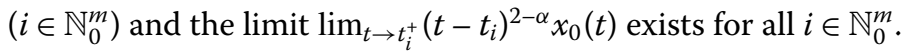

Similarly there exist $y_{0, i}, z_{0, i}:(0,1] \rightarrow \mathbb{R}$ such that

$$
\begin{aligned}
& \sup _{t \in\left[t_{i}, t_{i+1}\right]}\left|\left(t-t_{i}\right)^{2+\delta-\alpha} D_{0^{+}}^{\delta} x_{u, i}(t)-y_{0, i}(t)\right| \rightarrow 0, \quad u \rightarrow+\infty, i \in \mathbb{N}_{0}^{m}, \\
& \sup _{t \in\left[t_{i}, t_{i+1}\right]}\left|D_{0^{+}}^{\alpha-1} x_{u, i}(t)-z_{0, i}(t)\right| \rightarrow 0, \quad u \rightarrow+\infty, i \in \mathbb{N}_{0}^{m} .
\end{aligned}
$$

Let $y_{0}(t)=\left(t-t_{i}\right)^{\alpha-\delta-2} y_{0, i}(t)$ and $z_{0}(t)=z_{0, i}(t)$ for $t \in\left(t_{i}, t_{i+1}\right]\left(i \in \mathbb{N}_{0}^{m}\right)$. Then $y_{0}, z_{0} \in$ $C^{0}\left(t_{i}, t_{i+1}\right]\left(i \in \mathbb{N}_{0}^{m}\right)$ and the limits $\lim _{t \rightarrow t_{i}^{+}}\left(t-t_{i}\right)^{2+\delta-\alpha} y_{0}(t)$ and $\lim _{t \rightarrow t_{i}^{+}} z_{0}(t)$ exist for all $i \in \mathbb{N}_{0}^{m}$. 
Furthermore, using Lemma 2.2, for $t \in\left(t_{i}, t_{i+1}\right]$ there exists $c_{u j} \in \mathbb{R}$ such that

$$
\begin{aligned}
& \left|x_{u}(t)+\sum_{j=0}^{i} c_{u j}\left(t-t_{j}\right)^{\delta-1}-I_{0^{+}}^{\delta} y_{0}(t)\right| \\
& =\left|I_{0^{+}}^{\delta} D_{0^{+}}^{\delta} x_{u}(t)-I_{0^{+}}^{\delta} y_{0}(t)\right| \\
& \leq \int_{0}^{t} \frac{(t-s)^{\delta-1}}{\Gamma(\delta)}\left|D_{0^{+}}^{\delta} x_{u}(s)-y_{0}(s)\right| d s \\
& =\sum_{j=0}^{i-1} \int_{t_{j}}^{t_{j+1}} \frac{(t-s)^{\delta-1}}{\Gamma(\delta)}\left(s-t_{j}\right)^{\alpha-\delta-2}\left|\left(s-t_{j}\right)^{2+\delta-\alpha} D_{0^{+}}^{\delta} x_{u}(s)-\left(s-t_{j}\right)^{2+\delta-\alpha} y_{0}(s)\right| d s \\
& +\int_{t_{i}}^{t} \frac{(t-s)^{\delta-1}}{\Gamma(\delta)}\left(s-t_{i}\right)^{\alpha-\delta-2}\left|\left(s-t_{i}\right)^{2+\delta-\alpha} D_{0^{+}}^{\delta} x_{u}(s)-\left(s-t_{i}\right)^{2+\delta-\alpha} y_{0}(s)\right| d s \\
& =\sum_{j=0}^{i-1} \int_{t_{j}}^{t_{j+1}} \frac{(t-s)^{\delta-1}}{\Gamma(\delta)}\left(s-t_{j}\right)^{\alpha-\delta-2}\left|\left(s-t_{j}\right)^{2+\delta-\alpha} D_{0^{+}}^{\delta} x_{u}(s)-y_{0, j}(s)\right| d s \\
& +\int_{t_{i}}^{t} \frac{(t-s)^{\delta-1}}{\Gamma(\delta)}\left(s-t_{i}\right)^{\alpha-\delta-2}\left|\left(s-t_{i}\right)^{2+\delta-\alpha} D_{0^{+}}^{\delta} x_{u}(s)-y_{0, i}(s)\right| d s \\
& \leq\left(\sum_{j=0}^{i-1} \int_{t_{j}}^{t_{j+1}} \frac{(t-s)^{\delta-1}}{\Gamma(\delta)}\left(s-t_{j}\right)^{\alpha-\delta-2} d s+\int_{t_{i}}^{t} \frac{(t-s)^{\delta-1}}{\Gamma(\delta)}\left(s-t_{i}\right)^{\alpha-\delta-2} d s\right) \\
& \times \max \left\{\sup _{t \in\left(t_{i}, t_{i+1}\right]}\left|\left(t-t_{i}\right)^{2+\delta-\alpha} D_{0^{+}}^{\delta} x_{u}(t)-y_{0, i}(t)\right|: i \in N_{0}\right\} \\
& =\left(\sum_{j=0}^{i-1}\left(t-t_{j}\right)^{\alpha-2} \int_{0}^{\frac{t_{j+1}-t_{j}}{t-t_{j}}} \frac{(1-w)^{\delta-1}}{\Gamma(\delta)} w^{\alpha-\delta-2} d w+\left(t-t_{i}\right)^{\alpha-2} \int_{0}^{1} \frac{(1-w)^{\delta-1}}{\Gamma(\delta)} w^{\alpha-\delta-2} d w\right) \\
& \times \max \left\{\sup _{t \in\left(t_{i}, t_{i+1}\right]}\left|\left(t-t_{i}\right)^{2+\delta-\alpha} D_{0^{+}}^{\delta} x_{u}(t)-y_{0, i}(t)\right|: i \in N_{0}\right\} \\
& \leq \frac{\mathbf{B}(\delta, \alpha-\delta-1)}{\Gamma(\delta)} \sum_{j=0}^{i}\left(t-t_{j}\right)^{\alpha-2} \max \left\{\sup _{t \in\left(t_{i}, t_{i+1}\right]}\left|\left(t-t_{i}\right)^{2+\delta-\alpha} D_{0^{+}}^{\delta} x_{u}(t)-y_{0, i}(t)\right|: i \in N_{0}\right\} \\
& \rightarrow 0 \quad \text { as } u \rightarrow+\infty \text {. }
\end{aligned}
$$

Then $\lim _{u \rightarrow+\infty}\left[x_{u}(t)+\sum_{j=0}^{i} c_{u j}\left(t-t_{j}\right)^{\delta-1}\right]=I_{0^{+}}^{\delta} y_{0}(t)$. So $\left(t-t_{i}\right)^{\alpha-2} x_{0, i}(t)+\sum_{j=0}^{i} c_{0 j}\left(t-t_{j}\right)^{\delta-1}=$ $I_{0^{+}}^{\delta} y_{0}(t)\left(i \in \mathbb{N}_{0}^{m}\right)$. It follows that $x_{0}(t)+\sum_{j=0}^{i} c_{0 j}\left(t-t_{j}\right)^{\delta-1}=I_{0^{+}}^{\delta} y_{0}(t)$. Thus $y_{0}(t)=D_{0^{+}}^{\delta} x_{0}(t)$ for $t \in\left(t_{i}, t_{i+1}\right]$.

We have similarly for $t \in\left(t_{i}, t_{i+1}\right]$

$$
\begin{aligned}
& \left|x_{u}(t)+\sum_{j=0}^{i} c_{u j}\left(t-t_{j}\right)^{\alpha-2}-I_{0^{+}}^{\alpha-1} z_{0}(t)\right| \\
& =\left|I_{0^{+}}^{\alpha-1} D_{0^{+}}^{\alpha-1} x_{u}(t)-I_{0^{+}}^{\alpha-1} z_{0}(t)\right| \\
& \leq \int_{0}^{t} \frac{(t-s)^{\alpha-2}}{\Gamma(\alpha-1)}\left|D_{0^{+}}^{\alpha-1} x_{u}(s)-z_{0}(s)\right| d s \\
& =\sum_{j=0}^{i-1} \int_{t_{j}}^{t_{j+1}} \frac{(t-s)^{\alpha-2}}{\Gamma(\alpha-1)}\left|D_{0^{+}}^{\alpha-1} x_{u, j}(s)-z_{0, j}(s)\right| d s
\end{aligned}
$$




$$
\begin{aligned}
& +\int_{t_{i}}^{t} \frac{(t-s)^{\alpha-2}}{\Gamma(\alpha-1)}\left|D_{0^{+}}^{\alpha-1} x_{u, i}(s)-z_{0, i}(s)\right| d s \\
\leq & \int_{0}^{t} \frac{(t-s)^{\alpha-2}}{\Gamma(\alpha-1)} d s \max \left\{\sup _{t \in\left(t_{i}, t_{i+1}\right]}\left|D_{0^{+}}^{\alpha-1} x_{u, i}(t)-z_{0, i}(t)\right|: i \in N_{0}\right\} \\
= & \frac{t^{\alpha-1}}{\Gamma(\alpha)} \max \left\{\sup _{\left.t \in t_{i}, t_{i+1}\right]}\left|D_{0^{+}}^{\alpha-1} x_{u, i}(t)-z_{0, i}(t)\right|: i \in N_{0}\right\} \rightarrow 0 \quad \text { as } u \rightarrow+\infty .
\end{aligned}
$$

Then $\lim _{u \rightarrow+\infty}\left[x_{u}(t)+\sum_{j=0}^{i} c_{u j}\left(t-t_{j}\right)^{\alpha-2}\right]=I_{0^{+}}^{\alpha-1} z_{0}(t)$. So $\left(t-t_{i}\right)^{\alpha-2} x_{0, i}(t)+\sum_{j=0}^{i} c_{0 j}\left(t-t_{j}\right)^{\alpha-2}=$ $I_{0^{+}}^{\alpha-1} z_{0}(t)\left(i \in \mathbb{N}_{0}^{m}\right)$. It follows that $x_{0}(t)+\sum_{j=0}^{i} c_{0 j}\left(t-t_{j}\right)^{\alpha-2}=I_{0^{+}}^{\alpha-1} z_{0}(t)$. Thus $z_{0}(t)=D_{0^{+}}^{\alpha-1} x_{0}(t)$ for $t \in\left(t_{i}, t_{i+1}\right]$.

From the above discussion, we know that $x_{u} \rightarrow x_{0}$ as $u \rightarrow+\infty$ in $X_{\delta, \alpha}$. It follows that $X_{\delta, \alpha}$ is a Banach space. The proof is complete.

Denote $E=X_{\delta, \alpha} \times X_{\theta, \beta}$. Define $\|(x, y)\| \max \left\{\|x\|_{\delta, \alpha},\|y\|_{\theta, \beta}\right\}$. Then $E$ is a Banach space. For $y \in X_{\theta, \beta}$ and $x \in X_{\delta, \alpha}$, denote

$$
\begin{aligned}
& F_{y}(t)=p(t) f\left(t, y(t), D_{0^{+}}^{\theta} y(t), D_{0^{+}}^{\beta-1} y(t)\right), \\
& G_{x}(t)=q(t) g\left(t, x(t), D_{0^{+}}^{\delta} x(t), D_{0^{+}}^{\alpha-1} x(t)\right), \\
& I_{1 y}\left(t_{i}\right)=I_{1}\left(t_{i}, y\left(t_{i}\right), D_{0^{+}}^{\theta} y\left(t_{i}\right), D_{0^{+}}^{\beta-1} y\left(t_{i}\right)\right), \\
& J_{1 y}\left(t_{i}\right)=J_{1}\left(t_{i}, y\left(t_{i}\right), D_{0^{+}}^{\theta} y\left(t_{i}\right), D_{0^{+}}^{\beta-1} y\left(t_{i}\right)\right), \\
& I_{2 x}\left(t_{i}\right)=I_{2}\left(t_{i}, x\left(t_{i}\right), D_{0^{+}}^{\delta} x\left(t_{i}\right), D_{0^{+}}^{\alpha-1} x\left(t_{i}\right)\right), \\
& J_{2 x}\left(t_{i}\right)=J_{2}\left(t_{i}, x\left(t_{i}\right), D_{0^{+}}^{\delta} x\left(t_{i}\right), D_{0^{+}}^{\alpha-1} x\left(t_{i}\right)\right) .
\end{aligned}
$$

Lemma 2.4 Suppose that (a)-(d) hold and $(x, y) \in E$. Then $(u, v) \in E$ is a solution of

$$
\left\{\begin{array}{l}
D_{0^{+}}^{\alpha} u(t)=p(t) f\left(t, y(t), D_{0^{+}}^{\theta} y(t), D_{0^{+}}^{\beta-1} y(t)\right), \quad t \in\left(t_{i}, t_{i+1}\right], i \in \mathbb{N}_{0}^{m}, \\
D_{0^{+}}^{\beta} v(t)=q(t) g\left(t, x(t), D_{0^{+}}^{\delta} x(t), D_{0^{+}}^{\alpha-1} x(t)\right), \quad t \in\left(t_{i}, t_{i+1}\right], i \in \mathbb{N}_{0}^{m}, \\
D_{0^{+}}^{\alpha-1} u(0)=0, \quad u(1)=0, \quad D_{0^{+}}^{\beta-1} v(0)=0, \quad v(1)=0, \\
\lim _{t \rightarrow t_{i}^{+}}\left(t-t_{i}\right)^{2-\alpha} u(t)=I_{1}\left(t_{i}, y\left(t_{i}\right), D_{0^{+}}^{\delta} y\left(t_{i}\right), D_{0^{+}}^{\beta-1} y\left(t_{i}\right)\right), \quad i \in \mathbb{N}_{1}^{m}, \\
\lim _{t \rightarrow t_{i}^{+}} D_{0^{+}}^{\alpha-1} u(t)-D_{0^{+}}^{\alpha-1} u\left(t_{i}\right)=J_{1}\left(t_{i}, y\left(t_{i}\right), D_{0^{+}}^{\delta} y\left(t_{i}\right), D_{0^{+}}^{\beta-1} y\left(t_{i}\right)\right), \quad i \in \mathbb{N}_{1}^{m}, \\
\lim _{t \rightarrow t_{i}^{+}}\left(t-t_{i}\right)^{2-\beta} v(t)=I_{1}\left(t_{i}, x\left(t_{i}\right), D_{0^{+}}^{\delta} x\left(t_{i}\right), D_{0^{+}}^{\alpha-1} x\left(t_{i}\right)\right), \quad i \in \mathbb{N}_{1}^{m}, \\
\lim _{t \rightarrow t_{i}^{+}} D_{0^{+}}^{\beta-1} v(t)-D_{0^{+}}^{\beta-1} v\left(t_{i}\right)=J_{2}\left(t_{i}, x\left(t_{i}\right), D_{0^{+}}^{\delta} x\left(t_{i}\right), D_{0^{+}}^{\alpha-1} x\left(t_{i}\right)\right), \quad i \in \mathbb{N}_{1}^{m},
\end{array}\right.
$$

if and only if

$$
\begin{aligned}
u(t)= & \int_{0}^{t} \frac{(t-s)^{\alpha-1}}{\Gamma(\alpha)} F_{y}(s) d s \\
& -\left[\int_{0}^{1} \frac{(1-s)^{\alpha-1}}{\Gamma(\alpha)} F_{y}(s) d s+\sum_{j=1}^{m} \frac{\left(1-t_{j}\right)^{\alpha-1}}{\Gamma(\alpha)} J_{1 y}\left(t_{j}\right)+\sum_{j=1}^{m}\left(1-t_{j}\right)^{\alpha-2} I_{1 y}\left(t_{j}\right)\right] t^{\alpha-2} \\
& +\sum_{j=1}^{i} I_{1 y}\left(t_{j}\right)\left(t-t_{j}\right)^{\alpha-2}+\sum_{j=1}^{i} \frac{J_{1 y}\left(t_{j}\right)}{\Gamma(\alpha)}\left(t-t_{j}\right)^{\alpha-1}, \quad t \in\left(t_{i}, t_{i+1}\right], i \in \mathbb{N}_{0}^{m},
\end{aligned}
$$


and

$$
\begin{aligned}
v(t)= & \int_{0}^{t} \frac{(t-s)^{\beta-1}}{\Gamma(\beta)} G_{x}(s) d s \\
& -\left[\int_{0}^{1} \frac{(1-s)^{\beta-1}}{\Gamma(\beta)} G_{x}(s) d s+\sum_{j=1}^{m} I_{2 x}\left(t_{j}\right)\left(1-t_{j}\right)^{\beta-2}+\sum_{j=1}^{m} \frac{J_{2 x}\left(t_{j}\right)}{\Gamma(\beta)}\left(1-t_{j}\right)^{\beta-1}\right] t^{\beta-2} \\
& +\sum_{j=1}^{i} I_{2 x}\left(t_{j}\right)\left(t-t_{j}\right)^{\beta-2}+\sum_{j=1}^{i} \frac{J_{2 x}\left(t_{j}\right)}{\Gamma(\beta)}\left(t-t_{j}\right)^{\beta-1}, \quad t \in\left(t_{i}, t_{i+1}\right], i \in \mathbb{N}_{0}^{m} .
\end{aligned}
$$

Proof From $x \in X$ and $y \in Y$, we see that there exists a constant $r>0$ such that $\|x\|=r<$ $+\infty$. Then there exist constants $M_{r, f}, M_{r, g}, M_{1, r, I}, M_{2, r, I}, M_{1, r, J}, M_{2, r, J} \geq 0$ such that

$$
\begin{aligned}
\left|F_{y}(t)\right| & =|p(t)|\left|f\left(t, y(t), D_{0^{+}}^{\theta} y(t), D_{0^{+}}^{\beta-1} y(t)\right)\right| \\
& =|p(t)|\left|f\left(t, \frac{\left(t-t_{i}\right)^{2-\beta} y(t)}{\left(t-t_{i}\right)^{2-\beta}}, \frac{\left(t-t_{i}\right)^{2+\theta-\beta} D_{0^{+}}^{\theta} y(t)}{\left(t-t_{i}\right)^{2+\theta-\beta}}, D_{0^{+}}^{\beta-1} x(t)\right)\right| \\
& \leq M_{r_{r} f} t^{k_{1}}(1-t)^{l_{1}}, \quad t \in\left(t_{i}, t_{i+1}\right), i \in \mathbb{N}_{0}^{m},
\end{aligned}
$$

and similarly

$$
\begin{aligned}
& \left|G_{x}(t)\right| \leq M_{r, g} t^{k_{2}}(1-t)^{l_{2}}, \quad t \in\left(t_{i}, t_{i+1}\right), i \in \mathbb{N}_{0}^{m}, \\
& \left|I_{1}\left(t_{i}, y\left(t_{i}\right), D_{0^{+}}^{\theta} y\left(t_{i}\right), D_{0^{+}}^{\beta-1} y\left(t_{i}\right)\right)\right| \leq M_{1, r, I}, \quad i \in \mathbb{N}_{1}^{m}, \\
& \left|I_{2}\left(t_{i}, x\left(t_{i}\right), D_{0^{+}}^{\delta} x\left(t_{i}\right), D_{0^{+}}^{\alpha-1} x\left(t_{i}\right)\right)\right| \leq M_{2, r, I}, \quad i \in \mathbb{N}_{1}^{m}, \\
& \left|J_{1}\left(t_{i}, y\left(t_{i}\right), D_{0^{+}}^{\theta} y\left(t_{i}\right), D_{0^{+}}^{\beta-1} y\left(t_{i}\right)\right)\right| \leq M_{1, r, J}, \quad i \in \mathbb{N}_{1}^{m}, \\
& \left|J_{2}\left(t_{i}, x\left(t_{i}\right), D_{0^{+}}^{\delta} x\left(t_{i}\right), D_{0^{+}}^{\alpha-1} x\left(t_{i}\right)\right)\right| \leq M_{2, r, J}, \quad i \in \mathbb{N}_{1}^{m} .
\end{aligned}
$$

Hence

$$
\begin{aligned}
\left|\int_{0}^{t} \frac{(t-s)^{\alpha-1}}{\Gamma(\alpha)} F_{y}(s) d s\right| & \leq \int_{0}^{t} \frac{(t-s)^{\alpha-1}}{\Gamma(\alpha)}\left|F_{y}(s)\right| d s \\
& \leq \int_{0}^{t} \frac{(t-s)^{\alpha-1}}{\Gamma(\alpha)} s^{k_{1}}(1-s)^{l_{1}} M_{r, f} d s \\
& \leq M_{r, f} \int_{0}^{t} \frac{(t-s)^{\alpha-1}}{\Gamma(\alpha)} s^{k_{1}}(t-s)^{l_{1}} d s \\
& =M_{r, f} t^{\alpha+l_{1}+k_{1}} \int_{0}^{1} \frac{(1-w)^{\alpha+l_{1}-1}}{\Gamma(\alpha)} w^{k_{1}} d w \\
& =M_{r, f} t^{\alpha+l_{1}+k_{1}} \frac{\mathbf{B}\left(\alpha+l_{1}, k_{1}+1\right)}{\Gamma(\alpha)}<\infty, \quad t \in(0,1] .
\end{aligned}
$$

This means $F_{y}$ is an $\alpha$-integral function. Similarly we get

$$
\left|\int_{0}^{t} F_{y}(s) d s\right| \leq M_{r, f} \mathbf{B}\left(l_{1}+1, k_{1}+1\right)<\infty, \quad t \in(0,1] .
$$


From $u \in X$ and (9) in Lemma 2.3, we know that there exist constants $A_{i}, B_{i}\left(i \in \mathbb{N}_{0}^{m}\right)$ such that

$$
\begin{aligned}
u(t) & =\int_{0}^{t} \frac{(t-s)^{\alpha-1}}{\Gamma(\alpha)} F_{y}(s) d s+\sum_{j=0}^{i}\left[A_{j}\left(t-t_{j}\right)^{\alpha-1}+B_{j}\left(t-t_{j}\right)^{\alpha-2}\right], \\
t & \in\left(t_{i}, t_{i+1}\right], i \in \mathbb{N}_{0}^{m} .
\end{aligned}
$$

So

$$
\begin{aligned}
D_{0^{+}}^{\delta} u(t)= & \int_{0}^{t} \frac{(t-s)^{\alpha-\delta-1}}{\Gamma(\alpha-\delta)} F_{y}(s) d s+\frac{\Gamma(\alpha)}{\Gamma(\alpha-\delta)} \sum_{j=0}^{i} A_{j}\left(t-t_{j}\right)^{\alpha-\delta-1} \\
& +\frac{\Gamma(\alpha-1)}{\Gamma(\alpha-\delta-1)} \sum_{j=0}^{i} B_{j}\left(t-t_{j}\right)^{\alpha-\delta-2}, \quad t \in\left(t_{i}, t_{i+1}\right], i \in \mathbb{N}_{0}^{m}
\end{aligned}
$$

and

$$
D_{0^{+}}^{\alpha-1} u(t)=\int_{0}^{t} F_{y}(s) d s+\Gamma(\alpha) \sum_{j=0}^{i} A_{j}, \quad t \in\left(t_{i}, t_{i+1}\right], i \in \mathbb{N}_{0}^{m} .
$$

From $D_{0^{+}}^{\alpha-1} u(0)=0$ and $u(1)=0$ imply that $A_{0}=0$ and

$$
\int_{0}^{1} \frac{(1-s)^{\alpha-1}}{\Gamma(\alpha)} F_{y}(s) d s+\sum_{j=0}^{m}\left[A_{j}\left(1-t_{j}\right)^{\alpha-1}+B_{j}\left(1-t_{j}\right)^{\alpha-2}\right]=0 .
$$

Now we get by using the impulse conditions

$$
\begin{aligned}
& B_{i}=I_{1}\left(t_{i}, y\left(t_{i}\right), D_{0^{+}}^{\theta} y\left(t_{i}\right), D_{0^{+}}^{\beta-1} y\left(t_{i}\right)\right), \quad i \in \mathbb{N}_{1}^{m}, \\
& \Gamma(\alpha) A_{i}=J_{1}\left(t_{i}, y\left(t_{i}\right), D_{0^{+}}^{\theta} y\left(t_{i}\right), D_{0^{+}}^{\beta-1} y\left(t_{i}\right)\right), \quad i \in \mathbb{N}_{1}^{m} .
\end{aligned}
$$

Together with (21), we obtain

$$
B_{0}=-\int_{0}^{1} \frac{(1-s)^{\alpha-1}}{\Gamma(\alpha)} F_{y}(s) d s-\sum_{j=1}^{m}\left[\frac{\left(1-t_{j}\right)^{\alpha-1}}{\Gamma(\alpha)} J_{1 y}\left(t_{j}\right)+\left(1-t_{j}\right)^{\alpha-2} I_{1 y}\left(t_{j}\right)\right] .
$$

Substitute $A_{i}, B_{i}$ into (19), we get (14). Similarly we get (15).

Now we suppose that $u$ satisfies (14) and $v$ satisfies (15). We will prove that $u \in X$ and $v \in Y, u, v$ is a solution of BVP (13).

It is easy to see that $u \in X, v \in Y$. Furthermore, by direct computation, we get

$$
\begin{array}{ll}
D_{0^{+}}^{\alpha} u(t)=F_{y}(t), & t \in\left(t_{i}, t_{i+1}\right], i \in \mathbb{N}_{0}^{m}, \\
D_{0^{+}}^{\alpha-1} u(0)=0, & u(1)=0, \\
D_{0^{+}}^{\beta} \nu(t)=G_{x}(t), & t \in\left(t_{i}, t_{i+1}\right], i \in \mathbb{N}_{0}^{m}, \\
D_{0^{+}}^{\beta-1} v(0)=0, & v(1)=0, \\
\lim _{t \rightarrow t_{i}^{+}}\left(t-t_{i}\right)^{2-\alpha} u(t)=I_{1 y}\left(t_{i}\right), \quad i \in \mathbb{N}_{1}^{m},
\end{array}
$$




$$
\begin{aligned}
& \lim _{t \rightarrow t_{i}^{+}} D_{0^{+}}^{\alpha-1} u(t)-D_{0^{+}}^{\alpha-1} u\left(t_{i}\right)=J_{1 y}\left(t_{i}\right), \quad i \in \mathbb{N}_{1}^{m}, \\
& \lim _{t \rightarrow t_{i}^{+}}\left(t-t_{i}\right)^{2-\beta} v(t)=I_{2 y}\left(t_{i}\right), \quad i \in \mathbb{N}_{1}^{m}, \\
& \lim _{t \rightarrow t_{i}^{+}} D_{0^{+}}^{\beta-1} v(t)-D_{0^{+}}^{\beta-1} v\left(t_{i}\right)=J_{2 y}\left(t_{i}\right), \quad i \in \mathbb{N}_{1}^{m} .
\end{aligned}
$$

Then $(u, v)$ is a solution of BVP $(13)$. The proof is completed.

For $(x, y) \in E$, define $T(x, y)$ by $T(x, y)(t)=\left(\left(T_{1} y\right)(t),\left(T_{2} x\right)(t)\right)$ with

$$
\begin{aligned}
\left(T_{1} y\right)(t)= & \int_{0}^{t} \frac{(t-s)^{\alpha-1}}{\Gamma(\alpha)} F_{y}(s) d s \\
& -\left[\int_{0}^{1} \frac{(1-s)^{\alpha-1}}{\Gamma(\alpha)} F_{y}(s) d s+\sum_{j=1}^{m} \frac{\left(1-t_{j}\right)^{\alpha-1}}{\Gamma(\alpha)} J_{1 y}\left(t_{j}\right)+\sum_{j=1}^{m}\left(1-t_{j}\right)^{\alpha-2} I_{1 y}\left(t_{j}\right)\right] t^{\alpha-2} \\
& +\sum_{j=1}^{i} I_{1 y}\left(t_{j}\right)\left(t-t_{j}\right)^{\alpha-2}+\sum_{j=1}^{i} \frac{J_{1 y}\left(t_{j}\right)}{\Gamma(\alpha)}\left(t-t_{j}\right)^{\alpha-1}, \quad t \in\left(t_{i}, t_{i+1}\right], i \in \mathbb{N}_{0}^{m}
\end{aligned}
$$

and

$$
\begin{aligned}
\left(T_{2} x\right)(t)= & \int_{0}^{t} \frac{(t-s)^{\beta-1}}{\Gamma(\beta)} G_{x}(s) d s \\
& -\left[\int_{0}^{1} \frac{(1-s)^{\beta-1}}{\Gamma(\beta)} G_{x}(s) d s+\sum_{j=1}^{m} I_{2 x}\left(t_{j}\right)\left(1-t_{j}\right)^{\beta-2}+\sum_{j=1}^{m} \frac{J_{2 x}\left(t_{j}\right)}{\Gamma(\beta)}\left(1-t_{j}\right)^{\beta-1}\right] t^{\beta-2} \\
& +\sum_{j=1}^{i} I_{2 x}\left(t_{j}\right)\left(t-t_{j}\right)^{\beta-2}+\sum_{j=1}^{i} \frac{J_{2 x}\left(t_{j}\right)}{\Gamma(\beta)}\left(t-t_{j}\right)^{\beta-1}, \quad t \in\left(t_{i}, t_{i+1}\right], i \in \mathbb{N}_{0}^{m} .
\end{aligned}
$$

Lemma 2.5 Suppose that (a)-(d) hold. Then $T: E \rightarrow E$ is well defined and is completely continuous, $(x, y)$ is a solution of BVP (6) if and only if $(x, y)=T(x, y)$.

Proof By Lemma 2.4, we know that $T_{1} y \in X$ and $T_{2} x \in Y$. Then $T: E \rightarrow E$ is well defined. It is easy to show from Lemma 2.4 that $(x, y)$ is a solution of BVP (6) if and only if $(x, y)=$ $T(x, y)$.

Now, we prove that $T$ is completely continuous. It suffices to prove that $T_{1}: Y \rightarrow X$ and $T_{2}: X \rightarrow Y$ are completely continuous. We divide the proof of completely continuous property of $T_{1}$ into four steps. Similarly we can prove the completely continuous property of $T_{2}$.

Step 1 . Prove that $T_{1}$ is continuous.

Let $y_{n} \in Y(n=0,1,2, \ldots)$ with $y_{n} \rightarrow y_{0}$ as $n \rightarrow+\infty$. We will prove that $T_{1} y_{n} \rightarrow T_{1} y_{0}$ as $n \rightarrow+\infty$. It is easy to show that there exists $r>0$ such that $\left\|y_{n}\right\| \leq r, n=0,1,2, \ldots$, and $\left\|y_{n}-y_{0}\right\| \rightarrow 0$ as $n \rightarrow+\infty$. Then there exist constants $M_{r, f}, M_{1, r, I}, M_{1, r, J} \geq 0$ such that

$$
\begin{aligned}
& \left|F_{y_{n}}(t)\right| \leq M_{r_{f}} t^{k_{1}}(1-t)^{l_{1}}, \quad t \in\left(t_{i}, t_{i+1}\right), i \in \mathbb{N}_{0}^{m} 0, n=0,1,2, \ldots, \\
& \left|I_{1}\left(t_{i}, y_{n}\left(t_{i}\right), D_{0^{+}}^{\theta} y_{n}\left(t_{i}\right), D_{0^{+}}^{\beta-1} y_{n}\left(t_{i}\right)\right)\right| \leq M_{1, r, I}, \quad i \in \mathbb{N}_{1}^{m}, n=0,1,2, \ldots \\
& \left|J_{1}\left(t_{i}, y_{n}\left(t_{i}\right), D_{0^{+}}^{\theta} y_{n}\left(t_{i}\right), D_{0^{+}}^{\beta-1} y_{n}\left(t_{i}\right)\right)\right| \leq M_{1, r, J}, \quad i \in \mathbb{N}_{1}^{m}, n=0,1,2, \ldots .
\end{aligned}
$$


Using the definition in (22), one sees for $t \in\left(t_{i}, t_{i+1}\right]$ that

$$
\begin{aligned}
& \left(t-t_{i}\right)^{2-\alpha}\left|\left(T_{1} y_{n}\right)(t)-\left(T_{1} y_{0}\right)(t)\right| \\
& \leq\left(t-t_{i}\right)^{2-\alpha} \int_{0}^{t} \frac{(t-s)^{\alpha-1}}{\Gamma(\alpha)}\left|F_{y_{n}}(s)-F_{y_{0}}(s)\right| d s \\
& +\left(t-t_{i}\right)^{2-\alpha}\left[\int_{0}^{1} \frac{(1-s)^{\alpha-1}}{\Gamma(\alpha)}\left|F_{y_{n}}(s)-F_{y_{0}}(s)\right| d s+\sum_{j=1}^{m}\left(1-t_{j}\right)^{\alpha-2}\left|I_{1 y_{n}}\left(t_{j}\right)-I_{1 y_{0}}\left(t_{j}\right)\right|\right. \\
& \left.+\sum_{j=1}^{m} \frac{\left(1-t_{j}\right)^{\alpha-1}}{\Gamma(\alpha)}\left|J_{1 y_{n}}\left(t_{j}\right)-J_{1 y_{0}}\left(t_{j}\right)\right|\right] t^{\alpha-2}+\left(t-t_{i}\right)^{2-\alpha} \sum_{j=1}^{i}\left(t-t_{j}\right)^{\alpha-2}\left|I_{1 y_{n}}\left(t_{j}\right)-I_{1 y_{0}}\left(t_{j}\right)\right| \\
& +\left(t-t_{i}\right)^{2-\alpha} \sum_{j=1}^{i} \frac{\left|J_{1 y_{n}}\left(t_{j}\right)-J_{1 y_{0}}\left(t_{j}\right)\right|}{\Gamma(\alpha)}\left(t-t_{j}\right)^{\alpha-1} \\
& \leq 2 M_{r, f}\left(t-t_{i}\right)^{2-\alpha} \int_{0}^{t} \frac{(t-s)^{\alpha-1}}{\Gamma(\alpha)} s^{k_{1}}(1-s)^{l_{1}} d s \\
& +2 M_{r, f} \int_{0}^{1} \frac{(1-s)^{\alpha-1}}{\Gamma(\alpha)} s^{k_{1}}(1-s)^{l_{1}} d s+2 M_{1, r, I} \sum_{j=1}^{m}\left(1-t_{j}\right)^{\alpha-2}+2 M_{1, r, J} \sum_{j=1}^{m} \frac{\left(1-t_{j}\right)^{\alpha-1}}{\Gamma(\alpha)} \\
& +2 m M_{1, r, I}+2 M_{1, r, J} \sum_{j=1}^{m} \frac{t_{j+1}-t_{j}}{\Gamma(\alpha)} \\
& \leq 2 M_{r, f}\left(t-t_{i}\right)^{2-\alpha} t^{\alpha+k_{1}+l_{1}} \int_{0}^{1} \frac{(1-w)^{\alpha+l_{1}-1}}{\Gamma(\alpha)} w^{k_{1}} d w \\
& +2 M_{r, f} \frac{\mathbf{B}\left(\alpha+l_{1}, k_{1}+1\right)}{\Gamma(\alpha)}+2 M_{1, r, I} \sum_{j=1}^{m}\left(1-t_{j}\right)^{\alpha-2}+2 M_{1, r, J} \sum_{j=1}^{m} \frac{\left(1-t_{j}\right)^{\alpha-1}}{\Gamma(\alpha)} \\
& +2 m M_{1, r, I}+2 M_{1, r, J} \sum_{j=1}^{m} \frac{t_{j+1}-t_{j}}{\Gamma(\alpha)} \\
& \leq 4 M_{r, f} \frac{\mathbf{B}\left(\alpha+l_{1}, k_{1}+1\right)}{\Gamma(\alpha)}+2 M_{1, r, I}\left(\sum_{j=1}^{m}\left(1-t_{j}\right)^{\alpha-2}+m\right) \\
& +2 M_{1, r, J} \sum_{j=1}^{m}\left(\frac{\left(1-t_{j}\right)^{\alpha-1}}{\Gamma(\alpha)}+\frac{t_{j+1}-t_{j}}{\Gamma(\alpha)}\right) .
\end{aligned}
$$

Similarly we can prove for $t \in\left(t_{i}, t_{i+1}\right]$ that

$$
\begin{aligned}
& \left(t-t_{i}\right)^{2+\delta-\alpha}\left|D_{0^{+}}^{\delta}\left(T_{1} y_{n}\right)(t)-D_{0^{+}}^{\delta}\left(T_{1} y_{0}\right)(t)\right| \\
& \leq 2 M_{r, f}\left(\frac{\mathbf{B}\left(\alpha+l_{1}-\delta, k_{1}+1\right)}{\Gamma(\alpha-\delta)}+\frac{\Gamma(\alpha-1)}{\Gamma(\alpha-\delta-1)} \frac{\mathbf{B}\left(\alpha+l_{1}, k_{1}+1\right)}{\Gamma(\alpha)}\right) \\
& +2 M_{1, r, J}\left(\frac{\Gamma(\alpha-1)}{\Gamma(\alpha-\delta-1)} \sum_{j=1}^{m} \frac{\left(1-t_{j}\right)^{\alpha-1}}{\Gamma(\alpha)}+\frac{\Gamma(\alpha)}{\Gamma(\alpha-\delta)} \sum_{j=1}^{m} \frac{t_{j+1}-t_{j}}{\Gamma(\alpha)}\right) \\
& +2 M_{1, r, I}\left(\frac{\Gamma(\alpha-1)}{\Gamma(\alpha-\delta-1)} \sum_{j=1}^{m}\left(1-t_{j}\right)^{\alpha-2}+\frac{\Gamma(\alpha-1)}{\Gamma(\alpha-\delta-1)} m\right)
\end{aligned}
$$


and

$$
\begin{aligned}
& \left|D_{0^{+}}^{\alpha-1}\left(T_{1} y_{n}\right)(t)-D_{0^{+}}^{\alpha-1}\left(T_{1} y_{0}\right)(t)\right| \\
& \quad \leq \int_{0}^{t}\left|F_{y_{n}}(s)-F_{y_{0}}(s)\right| d s+\sum_{j=1}^{i}\left|J_{1 y_{n}}\left(t_{j}\right)-J_{1 y_{0}}\left(t_{j}\right)\right| \leq 2 M_{r, f} \mathbf{B}\left(l_{1}+1, k_{1}+1\right)+2 m M_{1, r, J} .
\end{aligned}
$$

By Lebesgue's dominated convergence theorem, we can show that

$$
\begin{aligned}
& \lim _{n \rightarrow \infty} \sup _{t \in\left(t_{i}, t_{i+1}\right]}\left(t-t_{i}\right)^{2-\alpha}\left|\left(T x_{n}\right)(t)-\left(T x_{0}\right)(t)\right|=0, \\
& \lim _{n \rightarrow \infty} \sup _{t \in\left(t_{i}, t_{i+1}\right]}\left(t-t_{i}\right)^{2+\delta-\alpha}\left|D_{0^{+}}^{\delta}\left(T x_{n}\right)(t)-D_{0^{+}}^{\delta}\left(T x_{0}\right)(t)\right|=0, \\
& \lim _{n \rightarrow \infty} \sup _{t \in\left(t_{i}, t_{i+1}\right]}\left|D_{0^{+}}^{\alpha-1}\left(T x_{n}\right)(t)-D_{0^{+}}^{\alpha-1}\left(T x_{0}\right)(t)\right|=0 .
\end{aligned}
$$

Hence $\left\|T x_{n}-T x_{0}\right\| \rightarrow 0$ as $n \rightarrow \infty$. Then $T$ is continuous.

Let $\Omega_{1} \subseteq X$ and $\Omega_{2} \subseteq Y$ be bounded sets of $X$ and $Y$, respectively. Then there exists $r>0$ such that $\|x\|,\|y\| \leq r, x \in \Omega_{1}, y \in \Omega_{2}$. So there exist constants $M_{r, f}, M_{r, g}, M_{1, r, I}, M_{2, r, I}, M_{1, r, J}$, $M_{2, r, J} \geq 0$ such that (16) and (17) hold for all $x \in \Omega_{1}, y \in \Omega_{2}$.

Step 2. Prove that $\left\{T_{1} y: y \in \Omega\right\}$ is bounded.

We have similarly to Step 1 for $t \in\left(t_{i}, t_{i+1}\right]$

$$
\begin{gathered}
\left(t-t_{i}\right)^{2-\alpha}|(T y)(t)| \leq 2 M_{r, f} \frac{\mathbf{B}\left(\alpha+l_{1}, k_{1}+1\right)}{\Gamma(\alpha)}+M_{1, r, I}\left(\sum_{j=1}^{m}\left(1-t_{j}\right)^{\alpha-2}+m\right) \\
+M_{1, r, J} \sum_{j=1}^{m}\left(\frac{\left(1-t_{j}\right)^{\alpha-1}}{\Gamma(\alpha)}+\frac{t_{j+1}-t_{j}}{\Gamma(\alpha)}\right), \\
\left(t-t_{i}\right)^{2+\delta-\alpha}\left|D_{0^{+}}^{\delta}\left(T_{1} y\right)(t)\right| \\
\leq M_{r, f}\left(\frac{\mathbf{B}\left(\alpha+l_{1}-\delta, k_{1}+1\right)}{\Gamma(\alpha-\delta)}+\frac{\Gamma(\alpha-1)}{\Gamma(\alpha-\delta-1)} \frac{\mathbf{B}\left(\alpha+l_{1}, k_{1}+1\right)}{\Gamma(\alpha)}\right) \\
+M_{1, r, J}\left(\frac{\Gamma(\alpha-1)}{\Gamma(\alpha-\delta-1)} \sum_{j=1}^{m} \frac{\left(1-t_{j}\right)^{\alpha-1}}{\Gamma(\alpha)}+\frac{\Gamma(\alpha)}{\Gamma(\alpha-\delta)} \sum_{j=1}^{m} \frac{t_{j+1}-t_{j}}{\Gamma(\alpha)}\right) \\
+M_{1, r, I}\left(\frac{\Gamma(\alpha-1)}{\Gamma(\alpha-\delta-1)} \sum_{j=1}^{m}\left(1-t_{j}\right)^{\alpha-2}+\frac{\Gamma(\alpha-1)}{\Gamma(\alpha-\delta-1)} m\right) \\
\left|D_{0^{+}}^{\alpha-1}\left(T_{1} y\right)(t)\right| \leq M_{r, f} \mathbf{B}\left(l_{1}+1, k_{1}+1\right)+m M_{1, r, J} .
\end{gathered}
$$

It follows that there exists a constant $M>0$ such that $\left\|T_{1} y\right\| \leq M_{1}$ for all $y \in \Omega_{2}$. Similarly we see that there exists a constant $M_{1}>0$ such that $\left\|T_{2} x\right\| \leq M_{2}$ for all $x \in \Omega_{1}$. From the above discussion, we see that $\left\{T(x, y): x \in \Omega_{1}, y \in \Omega_{2}\right\}$ is bounded.

Step 3. Prove that

$$
\begin{aligned}
& \left\{t \rightarrow\left(t-t_{i}\right)^{2-\alpha}\left(T_{1} y\right)(t): y \in \Omega\right\}, \\
& \left\{t \rightarrow(t-t)^{2+\delta-\alpha} D_{0^{+}}^{\delta}\left(T_{1} y\right)(t): x \in \Omega\right\},
\end{aligned}
$$




$$
\left\{t \rightarrow D_{0^{+}}^{\alpha-1}\left(T_{1} y\right)(t): y \in \Omega\right\}
$$

are equi-continuous on each $\left(t_{i}, t_{i+1}\right]\left(i \in \mathbb{N}_{0}^{m}\right)$.

Since $T: X \rightarrow X$, we can take

$$
\begin{aligned}
& \left.\left(t-t_{i}\right)^{2-\alpha}(T x)(t)\right|_{t=t_{i}}=\lim t \rightarrow t_{i}^{+}\left(t-t_{i}\right)^{2-\alpha}(T x)(t), \\
& \left.\left(t-t_{i}\right)^{2+\delta-\alpha} D_{0^{+}}^{\delta}(T x)(t)\right|_{t=t_{i}}=\lim _{t \rightarrow t_{i}^{+}}\left(t-t_{i}\right)^{2+\delta-\alpha} D_{0^{+}}^{\delta}(T x)(t), \\
& \left.D_{0^{+}}^{\alpha-1}(T x)(t)\right|_{t=t_{i}}=\lim _{t \rightarrow t_{i}^{+}} D_{0^{+}}^{\alpha-1}(T x)(t) .
\end{aligned}
$$

Then

$$
\left(t-t_{i}\right)^{2-\alpha}(T x)(t),\left(t-t_{i}\right)^{2+\delta-\alpha} D_{0^{+}}^{\delta}(T x)(t), D_{0^{+}}^{\alpha-1}(T x)(t)
$$

are continuous on $\left[t_{i}, t_{i+1}\right]$ for each $x \in \Omega$. So

$$
t^{2-\alpha}(T x)(t), t^{2+\delta-\alpha} D_{0^{+}}^{\delta}(T x)(t), D_{0^{+}}^{\alpha-1}(T x)(t)
$$

are uniformly continuous, for any $\varepsilon>0$, there exists $\delta_{0}>0$, when $s_{1}, s_{2} \in\left[t_{i}, t_{i+1}\right],\left|s_{1}-s_{2}\right|<$ $\delta_{0}$, and $x \in \Omega$, we can get

$$
\begin{aligned}
& \left|\left(s_{1}-t_{i}\right)^{2-\alpha}(T x)\left(s_{1}\right)-\left(s_{2}-t_{i}\right)^{2-\alpha}(T x)\left(s_{2}\right)\right|<\varepsilon, \\
& \left|\left(s_{1}-t_{i}\right)^{2+\delta-\alpha} D_{0^{+}}^{\delta}(T x)\left(s_{1}\right)-\left(s_{2}-t_{i}\right)^{2-\alpha}(T x)\left(s_{2}\right)\right|<\varepsilon, \\
& \left|D_{0^{+}}^{\alpha-1}(T x)\left(s_{1}\right)-D_{0^{+}}^{\alpha-1}(T x)\left(s_{2}\right)\right|<\varepsilon .
\end{aligned}
$$

This shows us that

$$
\begin{aligned}
& \left\{t \rightarrow t^{2-\alpha}(T x)(t): x \in \Omega\right\}, \\
& \left\{t \rightarrow t^{2+\delta-\alpha} D_{0^{+}}^{\delta}(T x)(t): x \in \Omega\right\}, \\
& \left\{t \rightarrow D_{0^{+}}^{\alpha-1}(T x)(t): x \in \Omega\right\}
\end{aligned}
$$

are equi-continuous on any closed subinterval of $\left[t_{i}, t_{i+1}\right]\left(i \in \mathbb{N}_{0}^{m}\right)$.

It follows from Steps 1-3 that $T$ is completely continuous. The proof is ended.

\section{Main results}

In this section, we prove the main theorem. Suppose that $\sigma_{j}, \tau_{j} \geq 0(j=1,2,3)$ are constants. We need the following assumptions:

(H1) there exist nonnegative constants $A_{j}, B_{j}(j=1,2,3)$ and two functions $\phi_{0}, \psi_{0}$ such that $p \psi_{0}$ is an $\alpha$-well integrable function and $q \phi_{0}$ a $\beta$-well integrable function and

$$
\begin{aligned}
& \left|f\left(t, \frac{y_{1}}{\left(t-t_{i}\right)^{2-\beta}}, \frac{y_{2}}{\left(t-t_{i}\right)^{2-\beta+\theta}}, y_{3}\right)-\psi_{0}(t)\right| \\
& \quad \leq \sum_{j=1}^{3} A_{j}\left|y_{j}\right|^{\sigma_{j}}, \quad t \in\left(t_{i}, t_{i+1}\right], y_{j} \in R(j=1,2,3), i \in \mathbb{N}_{0}^{m} 0,
\end{aligned}
$$




$$
\begin{aligned}
& \left|g\left(t, \frac{x_{1}}{\left(t-t_{i}\right)^{2-\alpha}}, \frac{x_{2}}{\left(t-t_{i}\right)^{2-\alpha+\delta}}, x_{3}\right)-\phi_{0}(t)\right| \\
& \quad \leq \sum_{j=1}^{3} B_{j}\left|x_{j}\right|^{\tau_{j}}, \quad t \in\left(t_{i}, t_{i+1}\right], x_{j} \in R(j=1,2,3), i \in \mathbb{N}_{0}^{m},
\end{aligned}
$$

(H2) there exist constants $I_{1 i}, J_{1 i}, I_{2 i}, J_{2 i}(i \in N), C_{j}, D_{j} \geq 0(j=1,2,3)$ such that

$$
\begin{aligned}
& \left|I_{1}\left(t_{i}, \frac{y_{1}}{\left(t_{i}-t_{i-1}\right)^{2-\beta}}, \frac{y_{2}}{\left(t_{i}-t_{i-1}\right)^{2-\beta+\theta}}, y_{3}\right)-I_{1 i}\right| \leq \sum_{j=1}^{3} C_{j}\left|y_{j}\right|^{\sigma_{j},} \quad i \in \mathbb{N}_{1}^{m}, \\
& \left|J_{1}\left(t, \frac{y_{1}}{\left(t-t_{i}\right)^{2-\beta}}, \frac{y_{2}}{\left(t-t_{i}\right)^{2-\beta+\theta}}, y_{3}\right)-J_{1 i}\right| \leq \sum_{j=1}^{3} D_{j}\left|y_{j}\right|^{\sigma_{j}}, \quad i \in \mathbb{N}_{1}^{m}, \\
& \left|I_{2}\left(t_{i}, \frac{x_{1}}{\left(t_{i}-t_{i-1}\right)^{2-\alpha}}, \frac{x_{2}}{\left(t_{i}-t_{i-1}\right)^{2-\alpha+\delta}}, x_{3}\right)-I_{2 i}\right| \leq \sum_{j=1}^{3} E_{j}\left|x_{j}\right|^{\tau_{j}}, \quad i \in \mathbb{N}_{1}^{m}, \\
& \left|J_{2}\left(t, \frac{x_{1}}{\left(t-t_{i}\right)^{2-\alpha}}, \frac{x_{2}}{\left(t-t_{i}\right)^{2-\alpha+\delta}}, x_{3}\right)-J_{2 i}\right| \leq \sum_{j=1}^{3} F_{j}\left|x_{j}\right|^{\tau_{j}}, \quad i \in \mathbb{N}_{1}^{m} .
\end{aligned}
$$

Denote

$$
\begin{aligned}
\Phi(t)= & \int_{0}^{t} \frac{(t-s)^{\alpha-1}}{\Gamma(\alpha)} p(s) \psi_{0}(s) d s \\
& -\left[\int_{0}^{1} \frac{(1-s)^{\alpha-1}}{\Gamma(\alpha)} p(s) \psi_{0}(s) d s+\sum_{j=1}^{m} \frac{\left(1-t_{j}\right)^{\alpha-1}}{\Gamma(\alpha)} J_{1 j}+\sum_{j=1}^{m}\left(1-t_{j}\right)^{\alpha-2} I_{1 j}\right] t^{\alpha-2} \\
& +\sum_{j=1}^{i}\left(t-t_{j}\right)^{\alpha-2} I_{1 j}+\sum_{j=1}^{i} \frac{J_{1 j}}{\Gamma(\alpha)}\left(t-t_{j}\right)^{\alpha-1}, \quad t \in\left(t_{i}, t_{i+1}\right], i \in \mathbb{N}_{0}^{m},
\end{aligned}
$$

and

$$
\begin{aligned}
\Psi(t)= & \int_{0}^{t} \frac{(t-s)^{\beta-1}}{\Gamma(\beta)} q(s) \phi_{0}(s) d s \\
& -\left[\int_{0}^{1} \frac{(1-s)^{\beta-1}}{\Gamma(\beta)} q(s) \phi_{0}(s) d s+\sum_{j=1}^{m} I_{2 j}\left(1-t_{j}\right)^{\beta-2}+\sum_{j=1}^{m} \frac{J_{2 j}}{\Gamma(\beta)}\left(1-t_{j}\right)^{\beta-1}\right] t^{\beta-2} \\
& +\sum_{j=1}^{i} I_{2 j}\left(t-t_{j}\right)^{\beta-2}+\sum_{j=1}^{i} \frac{J_{2 j}}{\Gamma(\beta)}\left(t-t_{j}\right)^{\beta-1}, \quad t \in\left(t_{i}, t_{i+1}\right], i \in \mathbb{N}_{0}^{m} .
\end{aligned}
$$

Denote

$$
\begin{aligned}
P_{1}= & \frac{2 \mathbf{B}\left(\alpha+l_{1}, k_{1}+1\right)}{\Gamma(\alpha)}+\frac{\mathbf{B}\left(\alpha-\delta+l_{1}, k_{1}+1\right)}{\Gamma(\alpha-\delta)}+\frac{\Gamma(\alpha-1)}{\Gamma(\alpha-\delta-1)} \frac{\mathbf{B}\left(\alpha+l_{1}, k_{1}+1\right)}{\Gamma(\alpha)} \\
& +\mathbf{B}\left(l_{1}+1, k_{1}+1\right), \\
P_{2}= & m+\sum_{j=1}^{m}\left(1-t_{j}\right)^{\alpha-2}+\frac{\Gamma(\alpha-1)}{\Gamma(\alpha-\delta-1)}\left(m+\sum_{j=1}^{m}\left(1-t_{j}\right)^{\alpha-2}\right), \\
P_{3}= & \frac{2 m}{\Gamma(\alpha)}+m\left(\frac{1}{\Gamma(\alpha-\delta)}+\frac{\Gamma(\alpha-1)}{\Gamma(\alpha) \Gamma(\alpha-\delta-1)}\right)+m,
\end{aligned}
$$


and

$$
\begin{aligned}
Q_{1}= & \frac{2 \mathbf{B}\left(\beta+l_{2}, k_{2}+1\right)}{\Gamma(\beta)}+\frac{\mathbf{B}\left(\beta-\theta+l_{2}, k_{2}+1\right)}{\Gamma(\beta-\theta)}+\frac{\Gamma(\beta-1)}{\Gamma(\beta-\theta-1)} \frac{\mathbf{B}\left(\beta+l_{2}, k_{2}+1\right)}{\Gamma(\beta)} \\
& +\mathbf{B}\left(l_{2}+1, k_{2}+1\right), \\
Q_{2}= & m+\sum_{j=1}^{m}\left(1-t_{j}\right)^{\beta-2}+\frac{\Gamma(\beta-1)}{\Gamma(\beta-\theta-1)}\left(m+\sum_{j=1}^{m}\left(1-t_{j}\right)^{\beta-2}\right), \\
Q_{3}= & \frac{2 m}{\Gamma(\beta)}+m\left(\frac{1}{\Gamma(\beta-\theta)}+\frac{\Gamma(\beta-1)}{\Gamma(\beta) \Gamma(\beta-\theta-1)}\right)+m, \\
\overline{P_{j}}= & P_{1} A_{j}+P_{2} C_{j}+P_{3} D_{j}, \quad \overline{Q_{j}}=Q_{1} B_{j}+Q_{2} E_{j}+Q_{3} F_{j} .
\end{aligned}
$$

Theorem 3.1 Let $\sigma=\max \left\{\sigma_{i}(i=1,2,3)\right\}$ and $\tau=\max \left\{\tau_{i}(i=1,2,3)\right\}$. Suppose that (a)-(d) and $(\mathrm{H} 1)-(\mathrm{H} 2)$ hold. Then BVP (6) has at least one solution if

(i) $\sigma \tau \in[0,1)$ or

(ii) $\sigma \tau=1$ with

$$
\begin{aligned}
& \sum_{j=1}^{3} Q_{j}\|\Phi\|^{\tau_{j}-\tau}<\left(\frac{1}{\sum_{j=1}^{3} P_{j}\|\Psi\|^{\sigma_{j}-\sigma}}\right)^{1 / \sigma} \text { or } \sum_{j=1}^{3} P_{j}\|\Psi\|^{\sigma_{j}-\sigma}<\left(\frac{1}{\sum_{j=1}^{3} Q_{j}\|\Phi\|^{\tau_{j}-\tau}}\right)^{1 / \tau} \\
& \text { or }
\end{aligned}
$$

(iii) $\sigma \tau>1$ with

$$
\begin{aligned}
& \frac{\sigma \tau-1}{\|\Psi\|} \sum_{j=1}^{3} Q_{j}\|\Phi\|^{\tau_{j}-\tau}\left[\|\Phi\|+\left(\frac{\sigma \tau\|\Psi\|}{\sigma \tau-1}\right)^{\sigma} \sum_{j=1}^{3} P_{j}\|\Psi\|^{\sigma_{j}-\sigma}\right]^{\tau} \leq 1, \quad \text { or } \\
& \frac{\sigma \tau-1}{\|\Phi\|} \sum_{j=1}^{3} P_{j}\|\Psi\|^{\sigma_{j}-\sigma}\left[\|\Psi\|+\left(\frac{\tau \sigma\|\Phi\|}{\sigma \tau-1}\right)^{\sigma} \sum_{j=1}^{3} Q_{j}\|\Phi\|^{\tau_{j}-\tau}\right]^{\sigma} \leq 1 .
\end{aligned}
$$

Proof It is easy to see that $(\Phi, \Psi) \in E$. For $r_{1}, r_{2}>0$, denote $\Omega_{r_{1}, r_{2}}=\{(x, y) \in E:\|x-\Phi\| \leq$ $\left.r_{1},\|y-\Psi\| \leq r_{2}\right\}$. One sees that $\|x\| \leq\|x-\Phi\|+\|\Phi\| \leq r_{1}+\|\Phi\|$ and $\|y\| \leq r_{2}+\|\Psi\|$ for all $(x, y) \in \Omega_{r_{1}, r_{2}}$.

Use (H1), for $(x, y) \in \Omega_{r_{1}, r_{2}}$, we have

$$
\begin{aligned}
& \left|f\left(t, y(t), D_{0^{+}}^{\theta} y(t), D_{0^{+}}^{\beta-1} y(t)\right)-\psi_{0}(t)\right| \\
& \quad=\left|f\left(t, \frac{\left(t-t_{i}\right)^{2-\beta} y(t)}{\left(t-t_{i}\right) t^{2-\beta}}, \frac{\left(t-t_{i}\right)^{2+\theta-\beta} D_{0^{+}}^{\theta} y(t)}{\left(t-t_{i}\right)^{2+\delta-\alpha}}, D_{0^{+}}^{\beta-1} y(t)\right)-\psi_{0}(t)\right| \\
& \quad \leq A_{1}\left|\left(t-t_{i}\right)^{2-\beta} y(t)\right|^{\sigma_{1}}+A_{2}\left|\left(t-t_{i}\right)^{2+\theta-\beta} D_{0^{+}}^{\theta} y(t)\right|^{\sigma_{2}}+A_{3}\left|D_{0^{+}}^{\beta-1} y(t)\right|^{\sigma_{3}} \\
& \quad \leq \sum_{j=1}^{3} A_{j}\left[r_{2}+\|\Psi\|\right]^{\sigma_{j}}, \quad t \in\left(t_{i}, t_{i+1}\right), i \in N_{0} .
\end{aligned}
$$

It follows that

$$
\left|f\left(t, y(t), D_{0^{+}}^{\theta} y(t), D_{0^{+}}^{\beta-1} y(t)\right)-\psi_{0}(t)\right| \leq \sum_{j=1}^{3} A_{j}\left[r_{2}+\|\Psi\|\right]^{\sigma_{j}} .
$$


Similarly using (H1)-(H2), we get for $(x, y) \in \Omega_{r}$

$$
\begin{aligned}
& \left|g\left(t, x(t), D_{0^{+}}^{\delta} x(t), D_{0^{+}}^{\alpha-1} x(t)\right)-\phi_{0}(t)\right| \leq \sum_{j=1}^{3} B_{j}\left[r_{1}+\|\Phi\|\right]^{\tau_{j},} \\
& \left|I_{1}\left(t_{i}, y\left(t_{i}\right), D_{0^{+}}^{\theta} y\left(t_{i}\right), D_{0^{+}}^{\beta-1} y\left(t_{i}\right)\right)-I_{1 i}\right| \leq \sum_{j=1}^{3} C_{j}\left[r_{2}+\|\Psi\|\right]^{\sigma_{j}}, \quad i \in \mathbb{N}_{1}^{m}, \\
& \left|J_{1}\left(t_{i}, y\left(t_{i}\right), D_{0^{+}}^{\theta} y\left(t_{i}\right), D_{0^{+}}^{\beta-1} y\left(t_{i}\right)\right)-J_{1 i}\right| \leq \sum_{j=1}^{3} D_{j}\left[r_{2}+\|\Psi\|\right]^{\sigma_{j},}, \quad i \in \mathbb{N}_{1}^{m}, \\
& \left|I_{2}\left(t_{i}, x\left(t_{i}\right), D_{0^{+}}^{\delta} x\left(t_{i}\right), D_{0^{+}}^{\alpha-1} x\left(t_{i}\right)\right)-I_{2 i}\right| \leq \sum_{j=1}^{3} E_{j}\left[r_{1}+\|\Phi\|\right]^{\tau_{j}}, \quad i \in \mathbb{N}_{1}^{m}, \\
& \left|J_{2}\left(t_{i}, x\left(t_{i}\right), D_{0^{+}}^{\delta} x\left(t_{i}\right), D_{0^{+}}^{\alpha-1} x\left(t_{i}\right)\right)-J_{2 i}\right| \leq \sum_{j=1}^{3} F_{j}\left[r_{1}+\|\Phi\|\right]^{\tau_{j}}, \quad i \in \mathbb{N}_{1}^{m} .
\end{aligned}
$$

Use (c) and (22), (25), (26), we get for $t \in\left(t_{i}, t_{i+1}\right]$

$$
\begin{aligned}
& \left(t-t_{i}\right)^{2-\alpha}\left|\left(T_{1} y\right)(t)-\Phi(t)\right| \\
& \leq\left(t-t_{i}\right)^{2-\alpha} \int_{0}^{t} \frac{(t-s)^{\alpha-1}}{\Gamma(\alpha)}\left|F_{y}(s)-p(s) \psi_{0}(s)\right| d s \\
& +\left(t-t_{i}\right)^{2-\alpha}\left[\int_{0}^{1} \frac{(1-s)^{\alpha-1}}{\Gamma(\alpha)}\left|F_{y}(s)-p(s) \psi_{0}(s)\right| d s+\sum_{j=1}^{m} \frac{\left(1-t_{j}\right)^{\alpha-1}}{\Gamma(\alpha)}\left|J_{1 y}\left(t_{j}\right)-J_{1 j}\right|\right. \\
& \left.+\sum_{j=1}^{m}\left(1-t_{j}\right)^{\alpha-2}\left|I_{1 y}\left(t_{j}\right)-I_{1 j}\right|\right] t^{\alpha-2} \\
& +\left(t-t_{i}\right)^{2-\alpha} \sum_{j=1}^{i}\left|I_{1 y}\left(t_{j}\right)-I_{1 j}\right|\left(t-t_{j}\right)^{\alpha-2}+\left(t-t_{i}\right)^{2-\alpha} \sum_{j=1}^{i} \frac{\left|J_{1 y}\left(t_{j}\right)-J_{1 j}\right|}{\Gamma(\alpha)}\left(t-t_{j}\right)^{\alpha-1} \\
& \leq\left(t-t_{i}\right)^{2-\alpha} \int_{0}^{t} \frac{(t-s)^{\alpha-1}}{\Gamma(\alpha)} s^{k_{1}}(1-s)^{l_{1}} d s \sum_{j=1}^{3} A_{j}\left[r_{2}+\|\Psi\|\right]^{\sigma_{j}} \\
& +\left(t-t_{i}\right)^{2-\alpha}\left[\int_{0}^{1} \frac{(1-s)^{\alpha-1}}{\Gamma(\alpha)} s^{k_{1}}(1-s)^{l_{1}} d s \sum_{j=1}^{3} A_{j}\left[r_{2}+\|\Psi\|\right]^{\sigma_{j}}\right. \\
& +\sum_{j=1}^{m} \frac{\left(1-t_{j}\right)^{\alpha-1}}{\Gamma(\alpha)} \sum_{j=1}^{3} D_{j}\left[r_{2}+\|\Psi\|\right]^{\sigma_{j}} \\
& \left.+\sum_{j=1}^{m}\left(1-t_{j}\right)^{\alpha-2} \sum_{j=1}^{3} C_{j}\left[r_{2}+\|\Psi\|\right]^{\sigma_{j}}\right] t^{\alpha-2} \\
& +\left(t-t_{i}\right)^{2-\alpha} \sum_{j=1}^{i}\left(t-t_{j}\right)^{\alpha-2} \sum_{j=1}^{3} C_{j}\left[r_{2}+\|\Psi\|\right]^{\sigma_{j}} \\
& +\left(t-t_{i}\right)^{2-\alpha} \sum_{j=1}^{i} \frac{\left(t-t_{j}\right)^{\alpha-1}}{\Gamma(\alpha)} \sum_{j=1}^{3} D_{j}\left[r_{2}+\|\Psi\|\right]^{\sigma_{j}}
\end{aligned}
$$




$$
\begin{aligned}
\leq & \left(t-t_{i}\right)^{2-\alpha} \int_{0}^{t} \frac{(t-s)^{\alpha+l_{1}-1}}{\Gamma(\alpha)} s^{k_{1}} d s \sum_{j=1}^{3} A_{j}\left[r_{2}+\|\Psi\|\right]^{\sigma_{j}} \\
& +\int_{0}^{1} \frac{(1-s)^{\alpha+l_{1}-1}}{\Gamma(\alpha)} s^{k_{1}} d s \sum_{j=1}^{3} A_{j}\left[r_{2}+\|\Psi\|\right]^{\sigma_{j}} \\
& +\sum_{j=1}^{m} \frac{\left(1-t_{j}\right)^{\alpha-1}}{\Gamma(\alpha)} \sum_{j=1}^{3} D_{j}\left[r_{2}+\|\Psi\|\right]^{\sigma_{j}} \\
& +\sum_{j=1}^{m}\left(1-t_{j}\right)^{\alpha-2} \sum_{j=1}^{3} C_{j}\left[r_{2}+\|\Psi\|\right]^{\sigma_{j}}+i \sum_{j=1}^{3} C_{j}\left[r_{2}+\|\Psi\|\right]^{\sigma_{j}} \\
& +\sum_{j=1}^{i} \frac{t_{i+1}-t_{j}}{\Gamma(\alpha)} \sum_{j=1}^{3} D_{j}\left[r_{2}+\|\Psi\|\right]^{\sigma_{j}} \\
\leq & \frac{\mathbf{B}\left(\alpha+l_{1}, k_{1}+1\right)}{\Gamma(\alpha)} \sum_{j=1}^{3} A_{j}\left[r_{2}+\|\Psi\|\right]^{\sigma_{j}}+\frac{\mathbf{B}\left(\alpha+l_{1}, k_{1}+1\right)}{\Gamma(\alpha)} \sum_{j=1}^{3} A_{j}\left[r_{2}+\|\Psi\|\right]^{\sigma_{j}} \\
& +\frac{m}{\Gamma(\alpha)} \sum_{j=1}^{3} D_{j}\left[r_{2}+\|\Psi\|\right]^{\sigma_{j}}+\sum_{j=1}^{m}\left(1-t_{j}\right)^{\alpha-2} \sum_{j=1}^{3} C_{j}\left[r_{2}+\|\Psi\|\right]^{\sigma_{j}} \\
& +m \sum_{j=1}^{3} C_{j}[r+\|\Psi\|]^{\sigma_{j}}+\frac{m}{\Gamma(\alpha)} \sum_{j=1}^{3} D_{j}\left[r_{2}+\|\Psi\|\right]^{\sigma_{j}} \\
= & \sum_{j=1}^{3}\left[\frac{2 \mathbf{B}\left(\alpha+l_{1}, k_{1}+1\right)}{\Gamma(\alpha)} A_{j}+\left(m+\sum_{j=1}^{m}\left(1-t_{j}\right)^{\alpha-2}\right) C_{j}+\frac{2 m}{\Gamma(\alpha)} D_{j}\right]\left[r_{2}+\|\Psi\|\right]^{\sigma_{j}} .
\end{aligned}
$$

One has from (22)

$$
\begin{aligned}
D_{0^{+}}^{\delta}\left(T_{1} y\right)(t)= & \int_{0}^{t} \frac{(t-s)^{\alpha-\delta-1}}{\Gamma(\alpha-\delta)} F_{y}(s) d s+\frac{\Gamma(\alpha)}{\Gamma(\alpha-\delta)} \sum_{j=1}^{i} \frac{\left(t-t_{j}\right)^{\alpha-\delta-1}}{\Gamma(\alpha)} J_{1 y}\left(t_{j}\right) \\
& -\frac{\Gamma(\alpha-1)}{\Gamma(\alpha-\delta-1)} t^{\alpha-\delta-2} \\
& \times\left[\int_{0}^{1} \frac{(1-s)^{\alpha-1}}{\Gamma(\alpha)} F_{y}(s) d s+\sum_{j=1}^{m} \frac{\left(1-t_{j}\right)^{\alpha-1}}{\Gamma(\alpha)} J_{1 y}\left(t_{j}\right)+\sum_{j=1}^{m}\left(1-t_{j}\right)^{\alpha-2} I_{1 y}\left(t_{j}\right)\right] \\
& +\frac{\Gamma(\alpha-1)}{\Gamma(\alpha-\delta-1)} \sum_{j=1}^{i}\left(t-t_{j}\right)^{\alpha-\delta-2} I_{1 y}\left(t_{j}\right), \quad t \in\left(t_{i}, t_{i+1}\right], i \in \mathbb{N}_{0}^{m}, \\
D_{0^{+}}^{\alpha-1}\left(T_{1} y\right)(t)= & \int_{0}^{t} F_{y}(s) d s+\sum_{j=1}^{i} J_{1 y}\left(t_{j}\right), \quad t \in\left(t_{i}, t_{i+1}\right], i \in \mathbb{N}_{0}^{m} .
\end{aligned}
$$

Similarly we have

$$
\begin{aligned}
& \left(t-t_{i}\right)^{2+\delta-\alpha}\left|D_{0^{+}}^{\delta}\left(T_{1} y\right)(t)-D_{0^{+}}^{\delta} \Phi(t)\right| \\
& \quad \leq \sum_{j=1}^{3}\left[\left(\frac{\mathbf{B}\left(\alpha-\delta+l_{1}, k_{1}+1\right)}{\Gamma(\alpha-\delta)}+\frac{\Gamma(\alpha-1)}{\Gamma(\alpha-\delta-1)} \frac{\mathbf{B}\left(\alpha+l_{1}, k_{1}+1\right)}{\Gamma(\alpha)}\right) A_{j}\right.
\end{aligned}
$$




$$
\begin{aligned}
& +\frac{\Gamma(\alpha-1)}{\Gamma(\alpha-\delta-1)}\left(m+\sum_{j=1}^{m}\left(1-t_{j}\right)^{\alpha-2}\right) C_{j} \\
& \left.+m\left(\frac{1}{\Gamma(\alpha-\delta)}+\frac{\Gamma(\alpha-1)}{\Gamma(\alpha) \Gamma(\alpha-\delta-1)}\right) D_{j}\right]\left[r_{2}+\|\Psi\|\right]^{\sigma_{j}}
\end{aligned}
$$

and

$$
\left|D_{0^{+}}^{\alpha-1}\left(T_{1} y\right)(t)-D_{0^{+}}^{\alpha-1} \Phi(t)\right| \leq \sum_{j=1}^{3}\left[\mathbf{B}\left(l_{1}+1, k_{1}+1\right) A_{j}+m D_{j}\right]\left[r_{2}+\|\Psi\|\right]^{\sigma_{j}} .
$$

It follows that

$$
\begin{aligned}
\left\|T_{1} y-\Phi\right\| & \leq \sum_{j=1}^{3}\left(P_{1} A_{j}+P_{2} C_{j}+P_{3} D_{j}\right)\left[r_{2}+\|\Psi\|\right]^{\sigma_{j}} \\
& \leq\left[r_{2}+\|\Psi\|\right]^{\sigma} \sum_{j=1}^{3}\left(P_{1} A_{j}+P_{2} C_{j}+P_{3} D_{j}\right)\|\Psi\|^{\sigma_{j}-\sigma} .
\end{aligned}
$$

Similarly we can get

$$
\begin{aligned}
\left\|T_{2} x-\Psi\right\| & \leq \sum_{j=1}^{3}\left(Q_{1} B_{j}+Q_{2} E_{j}+Q_{3} F_{j}\right)\left[r_{1}+\|\Phi\|\right]^{\tau_{j}} \\
& \leq\left[r_{1}+\|\Phi\|\right]^{\tau} \sum_{j=1}^{3}\left(Q_{1} B_{j}+Q_{2} E_{j}+Q_{3} F_{j}\right)\|\Phi\|^{\tau_{j}-\tau} .
\end{aligned}
$$

From (27), (28), we will seek $r_{1}, r_{2}>0$ such that

$$
\begin{aligned}
& {\left[r_{2}+\|\Psi\|\right]^{\sigma} \sum_{j=1}^{3} \overline{P_{j}}\|\Psi\|^{\sigma_{j}-\sigma} \leq r_{1},} \\
& {\left[r_{1}+\|\Phi\|\right]^{\tau} \sum_{j=1}^{3} \overline{Q_{j}}\|\Phi\|^{\tau_{j}-\tau} \leq r_{2} .}
\end{aligned}
$$

Then one has $T \Omega_{r_{1}, r_{2}} \subseteq \Omega_{r_{1}, r_{2}}$. By Schauder's fixed point theorem, $T$ has at least one fixed point $(x, y) \in \Omega_{r_{1}, r_{2}}$ which is a solution of BVP (6). It suffices to get positive solutions of the following inequality:

$$
\left[r_{1}+\|\Phi\|\right]^{\tau} \sum_{j=1}^{3} Q_{j}\|\Phi\|^{\tau_{j}-\tau} \leq r_{2} \leq\left(\frac{r_{1}}{\sum_{j=1}^{3} P_{j}\|\Psi\|^{\sigma_{j}-\sigma}}\right)^{1 / \sigma}-\|\Psi\|
$$

or

$$
\left[r_{2}+\|\Psi\|\right]^{\sigma} \sum_{j=1}^{3} P_{j}\|\Psi\|^{\sigma_{j}-\sigma} \leq r_{1} \leq\left(\frac{r_{2}}{\sum_{j=1}^{3} Q_{j}\|\Phi\|^{\tau_{j}-\tau}}\right)^{1 / \tau}-\|\Phi\| .
$$

Case 1. $\sigma \tau<1$. 
It is easy to see that

$$
\left[r_{1}+\|\Phi\|\right]^{\tau} \sum_{j=1}^{3} \overline{Q_{j}}\|\Phi\|^{\tau_{j}-\tau} \leq\left(\frac{r_{1}}{\sum_{j=1}^{3} \overline{P_{j}}\|\Psi\|^{\sigma_{j}-\sigma}}\right)^{1 / \sigma}-\|\Psi\|
$$

has a positive solution $r_{1}>0$ sufficiently large. Choose $r_{2}$ satisfying

$$
\left[r_{1}+\|\Phi\|\right]^{\tau} \sum_{j=1}^{3} \overline{Q_{j}}\|\Phi\|^{\tau_{j}-\tau} \leq r_{2} \leq\left(\frac{r_{1}}{\sum_{j=1}^{3} \overline{P_{j}}\|\Psi\|^{\sigma_{j}-\sigma}}\right)^{1 / \sigma}-\|\Psi\| .
$$

Then (29) has positive solutions $r_{1}>0$ and $r_{2}>0$. Then $T(x, y) \in \Omega_{r_{1}, r_{2}}$ for $(x, y) \in \Omega_{r_{1}, r_{2}}$. By Schauder's fixed point theorem, $T$ has at least one fixed point $(x, y) \in \Omega_{r_{1}, r_{2}}$. Then $(x, y)$ is a solution of BVP (6).

Case 2.1. $\sigma \tau=1$ and $\sum_{j=1}^{3} \overline{Q_{j}}\|\Phi\|^{\tau_{j}-\tau}\left(\sum_{j=1}^{3} \overline{P_{j}}\|\Psi\|^{\sigma_{j}-\sigma}\right)^{1 / \sigma}<1$.

Since

$$
\lim _{r_{1} \rightarrow+\infty} \frac{\left[r_{1}+\|\Phi\|\right]^{\tau} \sum_{j=1}^{3} \overline{Q_{j}}\|\Phi\|^{\tau_{j}-\tau}}{\left(\frac{r_{1}}{\sum_{j=1}^{3} P_{j}\|\Psi\|^{\sigma_{j}-\sigma}}\right)^{1 / \sigma}-\|\Psi\|}=\frac{\sum_{j=1}^{3} Q_{j}\|\Phi\|^{\tau_{j}-\tau}}{\left(\frac{1}{\sum_{j=1}^{3} \overline{P_{j}}\|\Psi\|^{\sigma_{j}-\sigma}}\right)^{1 / \sigma}}<1,
$$

we know that there exists $r_{1}>0$ sufficiently large such that

$$
\left[r_{1}+\|\Phi\|\right]^{\tau} \sum_{j=1}^{3} \overline{Q_{j}}\|\Phi\|^{\tau_{j}-\tau} \leq\left(\frac{r_{1}}{\sum_{j=1}^{3} \overline{P_{j}}\|\Psi\|^{\sigma_{j}-\sigma}}\right)^{1 / \sigma}-\|\Psi\| .
$$

Choose $r_{2}$ satisfying

$$
\left[r_{1}+\|\Phi\|\right]^{\tau} \sum_{j=1}^{3} \overline{Q_{j}}\|\Phi\|^{\tau_{j}-\tau} \leq r_{2} \leq\left(\frac{r_{1}}{\sum_{j=1}^{3} \overline{P_{j}}\|\Psi\|^{\sigma_{j}-\sigma}}\right)^{1 / \sigma}-\|\Psi\| .
$$

Then (29) has a positive solution $r_{1}, r_{2}$. Then $T(x, y) \in \Omega_{r_{1}, r_{2}}$ for $(x, y) \in \Omega_{r_{1}, r_{2}}$. By Schauder's fixed point theorem, $T$ has at least one fixed point $(x, y) \in \Omega_{r_{1}, r_{2}}$. Then $(x, y)$ is a solution of BVP (6).

Case 2.2. $\sigma \tau=1$ and $\sum_{j=1}^{3} \overline{P_{j}}\|\Psi\|^{\sigma_{j}-\sigma}\left(\sum_{j=1}^{3} \overline{Q_{j}}\|\Phi\|^{\tau_{j}-\tau}\right)^{1 / \tau}<1$.

Similarly to Case 2.1, use (31), we get solutions of BVP (6) by using the Schauder fixed point theorem.

Case 3. $\sigma \tau>1$.

Choose

$$
r_{1}=\left(\frac{\sigma \tau\|\Psi\|}{\sigma \tau-1}\right)^{\sigma} \sum_{j=1}^{3} \overline{P_{j}}\|\Psi\|^{\sigma_{j}-\sigma} .
$$

Since

$$
\frac{\sigma \tau-1}{\|\Psi\|} \sum_{j=1}^{3} \overline{Q_{j}}\|\Phi\|^{\tau_{j}-\tau}\left[\|\Phi\|+\left(\frac{\sigma \tau\|\Psi\|}{\sigma \tau-1}\right)^{\sigma} \sum_{j=1}^{3} \overline{P_{j}}\|\Psi\|^{\sigma_{j}-\sigma}\right]^{\tau} \leq 1,
$$

we know that (32) has a positive solution $r_{1}>0$. Choose $r_{2}$ such that (33) holds. Then we have $T(x, y) \in \Omega_{r_{1}, r_{2}}$ for $(x, y) \in \Omega_{r_{1}, r_{2}}$. By Schauder's fixed point theorem, $T$ has at least one fixed point $(x, y) \in \Omega_{r_{1}, r_{2}}$. Then $(x, y)$ is a solution of BVP (6). 
If

$$
\frac{\sigma \tau-1}{\|\Phi\|} \sum_{j=1}^{3} \overline{P_{j}}\|\Psi\|^{\sigma_{j}-\sigma}\left[\|\Psi\|+\left(\frac{\tau \sigma\|\Phi\|}{\sigma \tau-1}\right)^{\sigma} \sum_{j=1}^{3} \overline{Q_{j}}\|\Phi\|^{\tau_{j}-\tau}\right]^{\sigma} \leq 1,
$$

we choose

$$
r_{2}=\left(\frac{\tau \sigma\|\Phi\|}{\sigma \tau-1}\right)^{\tau} \sum_{j=1}^{3} \overline{Q_{j}}\|\Phi\|^{\tau_{j}-\tau}
$$

It is easy to verify that $r_{2}$ satisfies

$$
\left[r_{2}+\|\Psi\|\right]^{\sigma} \sum_{j=1}^{3} \overline{P_{j}}\|\Psi\|^{\sigma_{j}-\sigma} \leq\left(\frac{r_{2}}{\sum_{j=1}^{3} \overline{Q_{j}}\|\Phi\|^{\tau_{j}-\tau}}\right)^{1 / \tau}-\|\Phi\| .
$$

Choose $r_{1}$ such that

$$
\left[r_{2}+\|\Psi\|\right]^{\sigma} \sum_{j=1}^{3} \overline{P_{j}}\|\Psi\|^{\sigma_{j}-\sigma} \leq r_{1} \leq\left(\frac{r_{2}}{\sum_{j=1}^{3} \overline{Q_{j}}\|\Phi\|^{\tau_{j}-\tau}}\right)^{1 / \tau}-\|\Phi\| .
$$

Then we have $T(x, y) \in \Omega_{r_{1}, r_{2}}$ for $(x, y) \in \Omega_{r_{1}, r_{2}}$. By Schauder's fixed point theorem, $T$ has at least one fixed point $(x, y) \in \Omega_{r_{1}, r_{2}}$. Then $(x, y)$ is a solution of BVP (6).

The proof of Theorem 3.1 is completed.

Theorem 3.2 Suppose that (a)-(d) hold and there exist constants $M_{f}, M_{g}, M_{I 1}, M_{I 1}, M_{I 2}$, $M_{J 2} \geq 0$ such that

$$
\begin{aligned}
& \left|f\left(t, \frac{y_{1}}{\left(t-t_{i}\right)^{2-\beta}}, \frac{y_{2}}{\left(t-t_{i}\right)^{2-\beta+\theta}}, y_{3}\right)\right| \leq M_{f}, \quad t \in\left(t_{i}, t_{i+1}\right), y_{j} \in R(j=1,2,3), i \in \mathbb{N}_{0}^{m}, \\
& \left|g\left(t, \frac{x_{1}}{\left(t-t_{i}\right)^{2-\alpha}}, \frac{x_{2}}{\left(t-t_{i}\right)^{2-\alpha+\delta}}, x_{3}\right)\right| \leq M_{g}, \quad t \in\left(t_{i}, t_{i+1}\right), x_{j} \in R(j=1,2,3), i \in \mathbb{N}_{0}^{m}, \\
& \left|I_{1}\left(t_{i}, \frac{y_{1}}{\left(t_{i}-t_{i-1}\right)^{2-\beta}}, \frac{y_{2}}{\left(t_{i}-t_{i-1}\right)^{2-\beta+\theta}}, y_{3}\right)\right| \leq M_{11}, \quad i \in \mathbb{N}_{1}^{m}, \\
& \left|J_{1}\left(t, \frac{y_{1}}{\left(t-t_{i}\right)^{2-\beta}}, \frac{y_{2}}{\left(t-t_{i}\right)^{2-\beta+\theta}}, y_{3}\right)\right| \leq M_{J 1}, \quad i \in \mathbb{N}_{1}^{m}, \\
& \left|I_{2}\left(t_{i}, \frac{x_{1}}{\left(t_{i}-t_{i-1}\right)^{2-\alpha}}, \frac{x_{2}}{\left(t_{i}-t_{i-1}\right)^{2-\alpha+\delta}}, x_{3}\right)\right| \leq M_{I 2}, \quad i \in \mathbb{N}_{1}^{m}, \\
& \left|J_{2}\left(t, \frac{x_{1}}{\left(t-t_{i}\right)^{2-\alpha}}, \frac{x_{2}}{\left(t-t_{i}\right)^{2-\alpha+\delta}}, x_{3}\right)\right| \leq M_{J 2}, \quad i \in \mathbb{N}_{1}^{m} .
\end{aligned}
$$

Then BVP (6) has at least one solution in $X \times Y$.

Proof In Theorem 3.1, choose $\phi_{0}(t)=\psi_{0}(t)=0, \sigma_{1}=\sigma_{2}=\sigma_{3}=\tau_{1}=\tau_{2}=\tau_{3}=0, A_{1}=M_{f}$, $B_{1}=M_{g}, C_{1}=M_{I 1}, D_{1}=M_{J 1}, E_{1}=M_{I 2}, F_{1}=M_{J 2}$, and $A_{2}=A_{3}=B_{2}=B_{3}=C_{2}=C_{3}=D_{2}=$ $D_{3}=E_{2}=E_{3}=F_{2}=F_{3}=0$. It is easy to see that (H1) and (H2) hold. We get Theorem 3.2 from Theorem 3.1. The proof is completed. 


\section{An example}

In this section, we present an example to illustrate main theorems.

Example 4.1 Consider the following boundary value problem for the impulsive multiterm fractional differential equation:

$$
\left\{\begin{aligned}
D_{0^{+}}^{\frac{3}{2}} u(t)= & t^{-\frac{1}{4}}(1-t)^{-\frac{1}{4}}\left(a_{0}+a_{1}\left(t-t_{i}\right)^{\frac{\tau}{3}}[v(t)]^{\tau}+a_{2}\left(t-t_{i}\right)^{\frac{2 \tau}{3}}\left[D_{0^{+}}^{\frac{1}{3}} v(t)\right]^{\tau}\right. \\
& \left.+a_{3}\left[D_{0^{+}}^{\frac{2}{3}} v(t)\right]^{\tau}\right), \quad t \in\left(t_{i}, t_{i+1}\right), i \in \mathbb{N}_{0}^{10}, \\
D_{0^{+}}^{\frac{5}{3}} v(t)= & t^{-\frac{1}{6}}(1-t)^{-\frac{1}{6}}\left(b_{0}+b_{1}\left(t-t_{i}\right)^{\frac{\sigma}{2}}[u(t)]^{\sigma}+b_{2}\left(t-t_{i}\right)^{\frac{5 \sigma}{8}}\left[D_{0^{+}}^{\frac{1}{8}} u(t)\right]^{\sigma}\right. \\
& \left.+b_{3}\left[D_{0^{+}}^{\frac{1}{2}} u(t)\right]^{\sigma}\right), \quad t \in\left(t_{i}, t_{i+1}\right), i \in \mathbb{N}_{0}^{10}, \\
D_{0^{+}}^{\frac{1}{2}} u(0)= & 0, \quad u(1)=0, \quad D_{0^{+}}^{\frac{2}{3}} v(0)=0, \quad v(1)=0, \\
\lim _{t \rightarrow t_{i}^{+}}\left(t-t_{i}\right)^{\frac{1}{2}} u(t)=I_{1 i}, & \lim _{t \rightarrow t_{i}^{+}} D_{0^{+}}^{\frac{1}{2}} u(t)=J_{1 i}, \quad i \in \mathbb{N}_{1}^{10}, \\
\lim _{t \rightarrow t_{i}^{+}}\left(t-t_{i}\right)^{\frac{1}{2}} u(t)=I_{2 i}, & \lim _{t \rightarrow t_{i}^{+}} D_{0^{+}}^{\frac{1}{2}} u(t)=J_{2 i}, \quad i \in \mathbb{N}_{1}^{10},
\end{aligned}\right.
$$

where $a_{i}, b_{i} \in \mathbb{R}(i=0,1,2,3), I_{1 i}, I_{2 i}, J_{1 i}, J_{2 i} \in \mathbb{R}\left(i \in \mathbb{N}_{1}^{m}\right)$ and $\sigma \geq 0, \tau \geq 0,0=t_{0}<t_{1}=\frac{1}{11}<$ $\cdots<t_{10}=\frac{1}{2}<t_{11}=1, \mathbb{N}_{0}^{10}=\{0,1,2, \ldots, 10\}$, and $\mathbb{N}_{1}^{10}=\{1,2, \ldots, 10\}$.

Corresponding to BVP (6), we see that $\alpha=\frac{3}{2}, \beta=\frac{5}{3}, \delta=\frac{1}{8}, \theta=\frac{1}{3}, p(t)=t^{-\frac{1}{4}}(1-t)^{-\frac{1}{4}}$, $q(t)=t^{-\frac{1}{6}}(1-t)^{-\frac{1}{6}}$, with $k_{1}=l_{1}=-\frac{1}{4}, k_{2}=l_{2}=-\frac{1}{6}$. We find that $2+k_{i}+l_{i}>0$ and

$$
\begin{aligned}
& f\left(t, y_{1}, y_{2}, y_{3}\right)=a_{0}+a_{1}\left(t-t_{i}\right)^{\frac{\tau}{3}} y_{1}^{\tau}+a_{2}\left(t-t_{i}\right)^{\frac{2 \tau}{3}} y_{2}^{\tau}+a_{3} y_{3}^{\tau}, \quad t \in\left(t_{i}, t_{i+1}\right], i \in \mathbb{N}_{0}^{10}, \\
& g\left(t, x_{1}, x_{2}, x_{3}\right)=b_{0}+b_{1}\left(t-t_{i}\right)^{\frac{\sigma}{2}} x_{1}^{\sigma}+b_{2}\left(t-t_{i}\right)^{\frac{5 \sigma}{8}} x_{2}^{\sigma}+b_{3} x_{3}^{\sigma}, \quad t \in\left(t_{i}, t_{i+1}\right], i \in \mathbb{N}_{0}^{10}, \\
& I_{1}\left(t_{i}, y_{1}, y_{2}, y_{3}\right)=I_{1}, \quad J_{1}\left(t_{i}, y_{1}, y_{2}, y_{3}\right)=J_{1}, \quad i \in \mathbb{N}_{1}^{10}, \\
& I_{2}\left(t_{i}, x_{1}, x_{2}, x_{3}\right)=I_{2}, \quad J_{2}\left(t_{i}, x_{1}, x_{2}, x_{3}\right)=J_{2}, \quad i \in \mathbb{N}_{1}^{10} .
\end{aligned}
$$

One sees that (a)-(d), (H1), (H2) hold with

$$
\begin{aligned}
& \psi_{0}(t)=a_{0}, \quad \phi_{0}(t)=b_{0}, \quad I_{1 i}=I_{1}, \quad J_{1 i}=J_{1}, \quad I_{2 i}=I_{2}, \quad J_{2 i}=J_{2}, \\
& A_{i}=\left|a_{i}\right|, \quad B_{i}=\left|b_{i}\right|, \quad i=1,2,3, \\
& C_{i}=D_{i}=E_{i}=F_{i}=0, \quad i=1,2,3, \\
& \sigma_{1}=\sigma_{2}=\sigma_{3}=\sigma=\tau, \quad \tau_{1}=\tau_{2}=\tau_{3}=\tau=\sigma .
\end{aligned}
$$

It is easy to get

$$
\begin{aligned}
\Phi(t)= & A_{0} \int_{0}^{t} \frac{(t-s)^{\frac{1}{2}}}{\Gamma(3 / 2)} s^{-\frac{1}{4}}(1-s)^{-\frac{1}{4}} d s \\
& -\left[A_{0} \int_{0}^{1} \frac{(1-s)^{\frac{1}{2}}}{\Gamma(3 / 2)} s^{-\frac{1}{4}}(1-s)^{-\frac{1}{4}} d s+\sum_{j=1}^{10} \frac{\left(1-t_{j}\right)^{\frac{1}{2}}}{\Gamma(3 / 2)} J_{1}+\sum_{j=1}^{10}\left(1-t_{j}\right)^{-\frac{1}{2}} I_{1}\right] t^{-\frac{1}{2}} \\
& +\sum_{j=1}^{i}\left(t-t_{j}\right)^{-\frac{1}{2}} I_{1}+\sum_{j=1}^{i} \frac{J_{1}}{\Gamma(3 / 2)}\left(t-t_{j}\right)^{\frac{1}{2}}, \quad t \in\left(t_{i}, t_{i+1}\right], i \in \mathbb{N}_{0}^{10},
\end{aligned}
$$


and

$$
\begin{aligned}
\Psi(t)= & B_{0} \int_{0}^{t} \frac{(t-s)^{\frac{2}{3}}}{\Gamma(5 / 3)} s^{-\frac{1}{6}}(1-s)^{-\frac{1}{6}} d s \\
& -\left[B_{0} \int_{0}^{1} \frac{(1-s)^{\frac{2}{3}}}{\Gamma(5 / 3)} s^{-\frac{1}{6}}(1-s)^{-\frac{1}{6}} d s+\sum_{j=1}^{10} I_{2}\left(1-t_{j}\right)^{-\frac{1}{3}}+\sum_{j=1}^{10} \frac{J_{2}}{\Gamma(5 / 3)}\left(1-t_{j}\right)^{\frac{2}{3}}\right] t^{-\frac{1}{3}} \\
& +\sum_{j=1}^{i} I_{2}\left(t-t_{j}\right)^{-\frac{1}{3}}+\sum_{j=1}^{i} \frac{J_{2}}{\Gamma(5 / 3)}\left(t-t_{j}\right)^{\frac{2}{3}}, \quad t \in\left(t_{i}, t_{i+1}\right], i \in \mathbb{N}_{0}^{m} .
\end{aligned}
$$

By direct computation, using Mathlab 7.0, we have

$$
\begin{aligned}
& P_{1}=\frac{2 \mathbf{B}(1 / 4,3 / 4)}{\Gamma(3 / 2)}+\frac{\mathbf{B}(1 / 8,3 / 4)}{\Gamma(11 / 8)}+\frac{\Gamma(1 / 2)}{\Gamma(3 / 8)} \frac{\mathbf{B}(5 / 4,3 / 4)}{\Gamma(3 / 2)}+\mathbf{B}(3 / 4,3 / 4)<22.2, \\
& Q_{1}=\frac{2 \mathbf{B}(3 / 2,5 / 6)}{\Gamma(5 / 3)}+\frac{\mathbf{B}(7 / 6,5 / 6)}{\Gamma(4 / 3)}+\frac{\Gamma(2 / 3)}{\Gamma(1 / 3)} \frac{\mathbf{B}(3 / 2,5 / 6)}{\Gamma(5 / 3)}+\mathbf{B}(5 / 6,5 / 6)<5.1, \\
& \overline{P_{j}}=P_{1} A_{j}=P_{1}\left|a_{j}\right| \leq 22.2\left|a_{j}\right|, \\
& \overline{Q_{j}}=Q_{1} B_{j}=Q_{1}\left|b_{j}\right| \leq 5.1\left|b_{j}\right| .
\end{aligned}
$$

One sees that

$$
5.1 \sum_{j=1}^{3}\left|b_{j}\right|\left(22.2 \sum_{j=1}^{3}\left|a_{j}\right|\right)^{1 / \sigma}<1 \quad \text { or } \quad 22.2 \sum_{j=1}^{3}\left|a_{j}\right|\left(5.1 \sum_{j=1}^{3}\left|b_{j}\right|\right)^{1 / \tau}<1
$$

implies

$$
\sum_{j=1}^{3} \overline{Q_{j}}\left(\sum_{j=1}^{3} \overline{P_{j}}\right)^{1 / \sigma}<1 \quad \text { or } \sum_{j=1}^{3} \overline{P_{j}}\left(\sum_{j=1}^{3} \overline{Q_{j}}\right)^{1 / \tau}<1, \quad \text { respectively. }
$$

Furthermore

$$
\begin{aligned}
& 5.1 \frac{\sigma \tau-1}{\|\Psi\|} \sum_{j=1}^{3}\left|b_{j}\right|\left[\|\Phi\|+22.2\left(\frac{\sigma \tau\|\Psi\|}{\sigma \tau-1}\right)^{\sigma} \sum_{j=1}^{3}\left|a_{j}\right|\right]^{\tau} \leq 1 \quad \text { or } \\
& 22.2 \frac{\sigma \tau-1}{\|\Phi\|} \sum_{j=1}^{3}\left|a_{j}\right|\left[\|\Psi\|+5.1\left(\frac{\tau \sigma\|\Phi\|}{\sigma \tau-1}\right)^{\sigma} \sum_{j=1}^{3}\left|b_{j}\right|\right]^{\sigma} \leq 1
\end{aligned}
$$

implies

$$
\begin{aligned}
& \frac{\sigma \tau-1}{\|\Psi\|} \sum_{j=1}^{3} \overline{Q_{j}}\left[\|\Phi\|+\left(\frac{\sigma \tau\|\Psi\|}{\sigma \tau-1}\right)^{\sigma} \sum_{j=1}^{3} \overline{P_{j}}\right]^{\tau} \leq 1 \quad \text { or } \\
& \frac{\sigma \tau-1}{\|\Phi\|} \sum_{j=1}^{3} \overline{P_{j}}\left[\|\Psi\|+\left(\frac{\tau \sigma\|\Phi\|}{\sigma \tau-1}\right)^{\sigma} \sum_{j=1}^{3} \overline{Q_{j}}\right]^{\sigma} \leq 1, \quad \text { respectively. }
\end{aligned}
$$

It follows from Theorem 3.1 that BVP (10) has at least one solution if

(i) $\sigma \tau \in[0,1)$ or 
(ii) $\sigma \tau=1$ with (35) or

(iii) $\sigma \tau>1$ with (37).

Remark 4.1 It is easy to see that BVP (34) has at least one solution for any $a_{i}, b_{i}(i=$ $0,1,2,3), I_{1 i}, J_{1 i}, I_{2 i}, J_{2 i}(i=1,2,3)$ if $\sigma \tau<1$. Since sufficiently small $\left|a_{i}\right|,\left|b_{i}\right|(i=1,2,3)$ and fixed $a_{0}, b_{0}, I_{1 i}, J_{1 i}, I_{2 i}, J_{2 i}(i=1,2,3)$ imply (35) holds, BVP (34) has at least one solution for any $a_{0}, b_{0}, I_{1 i}, J_{1 i}, I_{2 i}, J_{2 i}(i=1,2,3)$ and sufficiently small $\left|a_{i}\right|,\left|b_{i}\right|(i=1,2,3)$ if $\sigma \tau=1$. Since sufficiently small $\left|a_{i}\right|,\left|b_{i}\right|(i=1,2,3)$ and fixed $a_{0}, b_{0}, I_{1 i}, J_{1 i}, I_{2 i}, J_{2 i}(i=1,2,3)$ imply (37) holds, BVP (34) has at least one solution for any $a_{0}, b_{0}, I_{1 i}, J_{1 i}, I_{2 i}, J_{2 i}(i=1,2,3)$ and sufficiently small $\left|a_{i}\right|,\left|b_{i}\right|(i=1,2,3)$ if $\sigma \tau>1$.

\section{Competing interests}

The authors declare that they have no competing interests.

\section{Authors' contributions}

Each of the authors, Yang and Liu contributed to each part of this study equally and read and approved the final version of the manuscript.

\section{Author details}

${ }^{1}$ Department of Computer, Guangdong Police College, Guangzhou, 510230, P.R. China. ²Department of Mathematics, Guangdong University of Finance and Economics, Guangzhou, 510320, P.R. China.

\section{Acknowledgements}

The authors would like to thank the referees and the editors for their careful reading and some useful comments on improving the presentation of this paper.

Supported by the Natural Science Foundation of Guangdong province (No. S2011010001900) and Natural science research project for colleges and universities of Guangdong Province (No: 2014KTSCX126).

Received: 12 August 2015 Accepted: 4 December 2015 Published online: 30 December 2015

\section{References}

1. Podlubny, I: Geometric and physical interpretation of fractional integration and fractional differentiation. Dedicated to the 60th anniversary of Prof. Francesco Mainardi. Fract. Calc. Appl. Anal. 5, 367-386 (2002)

2. Podlubny, I, Heymans, N: Physical interpretation of initial conditions for fractional differential equations with Riemann-Liouville fractional derivatives. Rheol. Acta 45, 765-771 (2006)

3. Kilbas, AA, Marichev, OI, Samko, SG: Fractional Integral and Derivatives: Theory and Applications. Gordon and Breach, London (1993)

4. Mainardi, F: Fractional calculus: some basic problems in continuum and statistical mechanics. In: Carpinteri, A, Mainardi, F (eds.) Fractals and Fractional Calculus in Continuum Mechanics. Springer, New York (1997)

5. Podlubny, I: Fractional Differential Equations. Mathematics in Science and Engineering, vol. 198. Academic Press, San Diego (1999)

6. Goodrich, CS: Existence of a positive solution to systems of differential equations of fractional order. Comput. Math. Appl. 62, 1251-1268 (2011)

7. Kang, $\mathrm{S}$, Chen, H, Guo, J: Existence of positive solutions for a system of Caputo fractional difference equations depending on parameters. Adv. Differ. Equ. 2015, Article ID 138 (2015)

8. Liu, Y, He, T, Shi, H: Existence of positive solutions for Sturm-Liouville BVPs of singular fractional differential equations. Sci. Bull. "Politeh." Univ. Buchar., Ser. A, Appl. Math. Phys. 74, 93-108 (2012)

9. Liu, Y, Yang, $X$ : Resonant boundary value problems for singular multi-tern fractional differential equations. Differ. Equ. Appl. 5(3), 409-472 (2013)

10. Liu, Y: Global existence of solutions for a system of singular fractional differential equations with impulse effects. J. Appl. Math. Inform. 33(3-4), 327-342 (2015)

11. Liu, Y: Existence of solutions of a class of impulsive periodic type BVPs for singular fractional differential systems. Korean J. Math. 23(1), 205-230 (2015)

12. Liu, Y: New results on the existence of solutions of boundary value problems for singular fractional differential systems with impulse effects. Tbil. Math. J. 8(2), 1-22 (2015)

13. Liu, Y, Ahmad, B: A study of impulsive multiterm fractional differential equations with single and multiple base points and applications. Sci. World J. 2014, Article ID 194346 (2014)

14. Liu, Y, Yang, P: IVPs for singular multi-term fractional differential equations with multiple base points and applications. Appl. Math. 41(4), 361-384 (2014)

15. Li, Y, Zhang, H: Solvability for system of nonlinear singular differential equations with integral boundary conditions. Bound. Value Probl. 2014, Article ID 158 (2014)

16. Mawhin, J: Topological Degree Methods in Nonlinear Boundary Value Problems. NSFCBMS Regional Conference Series in Mathematics. Am. Math. Soc., Providence (1979)

17. Babakhani, A, Baleanu, D: Existence of positive solutions for a class of delay fractional differential equations with generalization to N-term. Abstr. Appl. Anal. 2011, Article ID 391971 (2011) 
18. Nyamoradi, N, Bashiri, T, Vaezpour, SM, Baleanu, D: Uniqueness and existence of positive solutions for singular fractional differential equations. Electron. J. Differ. Equ. 2014, Article ID 130 (2014)

19. Zhou, W, Chu, Y, Baleanu, D: Uniqueness and existence of positive solutions for a multi-point boundary value problem of singular fractional differential equations. Adv. Differ. Equ. 2013, Article ID 114 (2013)

20. Jleli, M, Samet, B: Existence of positive solutions to a coupled system of fractional differential equations. Math. Methods Appl. Sci. 38(6), 1014-1031 (2015)

21. Ntouyas, SK, Obaid, M: A coupled system of fractional differential equations with nonlocal integral boundary conditions. Adv. Differ. Equ. 2012, 130 (2012)

22. Shah, K, Khan, RA: Existence and uniqueness of positive solutions to a coupled system of nonlinear fractional order differential equations with anti periodic boundary conditions. Differ. Equ. Appl. 7(2), 245-262 (2015)

23. Sun, J, Liu, Y, Liu, G: Existence of solutions for fractional differential systems with anti-periodic boundary conditions. Comput. Math. Appl. 64, 1557-1566 (2012)

24. Sun, S, Li, Q, Li, Y: Existence and uniqueness of solutions for a coupled system of multi-term nonlinear fractional differential equations. Comput. Math. Appl. 64, 3310-3320 (2012)

25. Wang, Y, Liu, L, Wu, Y: Positive solutions for a class of higher-order singular semipositone fractional differential systems with coupled integral boundary conditions and parameters. Adv. Differ. Equ. 2014, Article ID 268 (2014)

26. Wang, J, Xiang, H, Liu, Z: Positive solution to nonzero boundary values problem for a coupled system of nonlinear fractional differential equations. Int. J. Differ. Equ. 2010, Article ID 186928 (2010)

27. Zhai, C, Hao, M: Multi-point boundary value problems for a coupled system of nonlinear fractional differential equations. Adv. Differ. Equ. 2015, Article ID 147 (2015)

28. Zhao, K, Gong, P: Positive solutions of Riemann-Stieltjes integral boundary problems for the nonlinear coupling system involving fractional-order derivatives. Adv. Differ. Equ. 2014, Article ID 254 (2014)

29. Su, W: Boundary value problem for a coupled system of nonlinear fractional differential equations. Appl. Math. Lett. 22(1), 64-69 (2009)

30. Yang, $\mathrm{A}, \mathrm{Ge}, \mathrm{W}$ : Positive solutions for boundary value problems of $\mathrm{N}$-dimension nonlinear fractional differential systems. Bound. Value Probl. 2008, Article ID 437453 (2008). doi:10.1155/2008/437453

31. Ahmad, B, Nieto, Jj: Existence results for a coupled system of nonlinear fractional differential equations with three-point boundary conditions. Comput. Math. Appl. 58, 1838-1843 (2009)

32. Zou, Y, Liu, L, Cui, Y: The existence of solutions for four-point coupled boundary value problems of fractional differential equations at resonance. Abstr. Appl. Anal. 2014, Article ID 314083 (2014)

33. Gaber, M, Brikaa, MG: Existence results for a coupled system of nonlinear fractional differential equation with four-point boundary conditions. ISRN Math. Anal. 2011, Article ID 468346 (2011)

34. Shah, K, Khalil, H, Khan, RA: Investigation of positive solution to a coupled system of impulsive boundary value problems for nonlinear fractional order differential equations. Chaos Solitons Fractals 77, 240-246 (2015)

35. Zhang, X, Zhu, C, Wu, Z: Solvability for a coupled system of fractional differential equations with impulses at resonance. Bound. Value Probl. 2013, Article ID 80 (2013)

\section{Submit your manuscript to a SpringerOpen ${ }^{\circ}$ journal and benefit from:}

- Convenient online submission

Rigorous peer review

- Immediate publication on acceptance

- Open access: articles freely available online

- High visibility within the field

- Retaining the copyright to your article 